\title{
The Effect of 3D Geometry on Unsteady Gust Response, Using a Vortex Lattice Model
}

\author{
A. S. M. Smyth* and A. M. Young ${ }^{\dagger}$ \\ Whittle laboratory, Cambridge University Engineering Department, United Kingdom \\ L. Di Mare ‡ $^{\ddagger}$ \\ Department of Engineering Science, University of Oxford, United Kingdom
}

\begin{abstract}
Unsteady flow response is an important consideration for a range of engineering applications, from unmanned air vehicles, where it has implications for control, to tidal turbines, where the accurate calculation of fatigue load is vital. Designers often use 2D strip-theory predictions for both steady and unsteady performance, applying Theodorsens unsteady transfer function for uniform gusts at each blade section to estimate the unsteady bending moments on the turbine blades. The purpose of this investigation is to explore the limits of the applicability of this $2 \mathrm{D}$ classical unsteady aerofoil theory to aerofoils with significant 3D geometry features. Using a harmonic vortex lattice model, this study shows that there are significant 3D features in the unsteady flow response, which increase with decreasing reduced frequency and with decreasing aspect ratio. The response near the blade tips is strongly $3 \mathrm{D}$, and does not reach the $2 \mathrm{D}$ characteristic, even at high frequencies. The phase response also varies strongly along the span, leading to different blade sections responding out of phase with each other even with no spanwise gust variation. This has significant implications for bending moment calculations, with require integration of the load along the span. The observed 3D effects are shown to be caused by changes to the spanwise component of the unsteady wake, and by the presence and behaviour of a streamwise unsteady wake. The results for a model tidal turbine geometry show that the Loewy function does not capture returning wake effects adequately, but that it does model the mid-span response characteristic well at reduced frequencies over 0.8 . The study concludes that using transfer functions from 2D classical aerofoil theory provides a conservative estimate of the blade loads affecting a tidal turbine, but only if no steady tip-loss corrections have been applied to the unsteady response. If tip-loss corrections are applied to the quasi-steady lift response before unsteady transfer functions are used, the resulting load amplitude will be significantly under-predicted.
\end{abstract}

\section{Introduction}

The problem of aerofoils encountering unsteady flow is common to many engineering applications, from helicopters and unmanned air vehicles to tidal turbines. In all these applications, the calculation of fatigue life, and the design of control algorithms, relies on accurate prediction of the effect of this unsteady flow on the aerofoil loading. Quasi-steady models are often insufficient, because they neglect important flow physics associated with the unsteady response. This study focuses in particular on the prediction of unsteady loads on horizontal-axis tidal turbines. The nature of tidal channel flows presents unique challenges to device designers due to the high level of unsteadiness, resulting in time-varying loads and, therefore, integrity and performance issues. Accurate estimates of fatigue loading and lifetime will prevent premature failures and over-cautious design, both of which are important to reduce costs and increase reliability for the nascent tidal industry.

*PhD student, Whittle Laboratory, 1 JJ Thomson Ave, Cambridge CB3 0DY, UK.

†Senior CDT fellow, Whittle Laboratory, 1 JJ Thomson Ave, Cambridge CB3 0DY, UK.

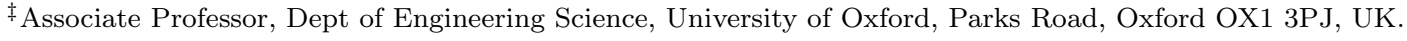


Tidal turbine designers use Blade Element Momentum Theory (BEMT) to calculate the steady-state performance and unsteady load response of new designs. In this strip-theory method, the blade is divided into annular sections which are assumed to be independent from each other. ${ }^{1}$ The $2 \mathrm{D}$ performance of each section is evaluated, and the results are interpolated along the span. Empirical and semi-empirical models are used to correct for the effect of three-dimensional flow on the steady performance (Prandtl's tip loss correction, for example). In order to capture unsteady effects, the state-of-the-art design software GH Tidal Bladed uses "classical" unsteady aerofoil theory to estimate the variation in load at each blade section. ${ }^{2}$ Classical theories assume that the flow can be approximated as inviscid flow over a 2D flat plate at zero mean angle of attack, which allows the derivation of analytical unsteady transfer functions (see Leishman ${ }^{3}$ for a summary). These unsteady transfer functions give the load variation for a given gust as a function of the quasi-steady 2D lift coefficient, and are readily applicable to BEMT methods. Examples of transfer functions include Theodorsen's function ${ }^{4}$ for uniform gusts and Sears' function ${ }^{5}$ for sinusoidal gusts.

The benefits of BEMT-based strip-theory methods are their inherent speed, combined with their ability to provide reasonable estimates for the steady turbine performance. ${ }^{6}$ Tidal turbines, however, generally have low aspect ratio blades with significant taper ratio, due to the high power density of the flow. As a result, the assumption of $2 \mathrm{D}$ flow made in BEMT is unlikely to apply. ${ }^{7}$ In addition to this, the unsteady gust environment found in tidal sites is strongly $3 \mathrm{D}$ in nature. ${ }^{8}$

Analysis equivalent to that of classical 2D aerofoil theory for 3D geometries is prohibitively complex, but some work has been done on deriving semi-analytical methods to evaluate 3D unsteady flow. Namba ${ }^{910}$ and Schulten ${ }^{11}$ both developed semi-analytical theories to calculate 3D unsteady load response in turbomachinery cascades, and evaluated the validity of strip-theory assumptions for such applications. They found that strip theory deviated strongly from the three-dimensional analysis especially at subsonic and transonic flow speeds, and that the unsteady response near the blade tip was poorly predicted. The 3D effects were found to increase with decreasing blade aspect ratio and decreasing reduced frequency. Other studies have also been carried out to predict 3D behaviour of aerofoils in unsteady flow, primarily for the prediction of flutter in turbomachinery cascades. ${ }^{121314}$ These studies have concluded that strip theory deviates from the 3D predictions, especially near the blade tips, but the physical causes are often unclear.

The focus of this study is on determining the significant 3D effects in the flow regime experienced by tidal turbines. The topic of unsteady load on tidal turbines has received some attention recently, ${ }^{151617}$ due to the continuing problems with fatigue life predictions. There is however limited understanding of 3D effects, and of how strip-theory estimates deviate from 3D predictions. This paper will address the following questions: When are 3D unsteady effects important? What are the primary sources of 3D unsteady load which must be modelled for accurate calculations? And, for a rotor or tidal turbine, what design parameters can affect the unsteady load?

To answer these questions, this study uses a vortex lattice model to analyse the unsteady response of several wing and rotor geometries and to assess the impact of different geometry parameters. The inviscid vortex lattice method is shown to capture the $2 \mathrm{D}$ unsteady load to the same level of accuracy as established classical unsteady aerofoil theory. In the absence of separated flow, the vortex lattice method can capture the load response of complex flow features and geometries. ${ }^{1819}$ Its intrinsic speed is a great advantage; a simulation that would take hours or days to complete using URANS or LES can be evaluated in seconds or minutes. This combination of speed and accuracy makes it a powerful tool for studying and comparing many geometries and flow states.

Sequeira ${ }^{20}$ mapped out the gust frequencies and amplitudes that a tidal turbine is likely to encounter during its lifetime. He estimated that the range of reduced frequencies experienced by a turbine was between 0.0 and 1.5. Most of the gust amplitudes fell within the attached flow region, although some rare, extreme flow occurrences would generate gust amplitudes in the aerofoil stall region. This study will therefore assume attached flow throughout, and will study the response characteristics in the reduced frequency range from 0 to 2.0. The limits of the applicability of $2 \mathrm{D}$ theory will be examined by applying the vortex lattice method to increasingly complex geometries, starting with a 2D flat plate and building in complexity to a model tidal turbine geometry. The response of geometries with three-dimensional features to two-dimensional gusts will 
be shown, with the aim of capturing the key features of 3D response. In many real flow environments, the gusts will also be strongly three-dimensional, but this is outside the scope of the present study, and the gusts examined will be uniform in the spanwise direction.

\section{Methods}

\section{II.A. Classical unsteady aerofoil theory}

The interaction of unsteady flow with a lifting surface results in two effects that must be accounted for when evaluating the lift response: the added mass and the unsteady wake. ${ }^{3}$ The added mass results from the fluid around the aerofoil being accelerated due to the transient flow movement, imparting a resultant force on the aerofoil proportional to the time rate-of-change of the aerofoil circulation. If the unsteady flow occurs with high frequency, this added mass force may become very large. The second effect, the unsteady wake, is generated to satisfy Kelvin's theorem of constant circulation in the flow. As the aerofoil interacts with the gust, its circulation changes and therefore a vortex of equal and opposite strength must be shed continuously. This wake induces a downwash velocity on the aerofoil, imparting unsteady aerodynamic damping on the aerofoil lift.

For harmonic gusts, the degree to which the load response is unsteady is determined by the reduced frequency. The reduced frequency is traditionally given with respect to the semichord, but in this study the recently more commonly used ${ }^{21}$ full chord version of reduced frequency is used:

$$
f_{\text {red }}=\frac{\omega * \text { chord }}{U}
$$

where $\omega=2 \pi f$ is the gust angular frequency. In this study the velocity $\mathrm{U}$ is taken as the local relative flow velocity (including rotational velocity) at each span position. The reduced frequency is a measure of how many chord lengths a fluid particle travels during a gust wavelength and has repeatedly been found to be the dominant parameter determining the properties of unsteady flow in $2 \mathrm{D}{ }^{6}$

In classical aerofoil theory, a number of simplifying assumptions are used to derive analytical transfer functions for the unsteady load response to a gust of a given amplitude and frequency. The aerofoil is modelled as a 2D flat plate at zero mean incidence, and the flow is treated as inviscid. The wake is assumed to leave the trailing edge along the horizontal, with the steady freestream velocity, and gust amplitudes are assumed to be small. ${ }^{5}$

Different gusts require different transfer functions, and the choice of appropriate transfer function for a given application is an important one for designers. Sequeira ${ }^{21}$ noted that the difference in loading given by two different transfer functions can generate an order of magnitude difference in the estimation of tidal turbine

fatigue life. The most commonly used transfer function for harmonic flow is the Theodorsen function, ${ }^{20}$ which models the effect of pitching and heaving aerofoils, and that of chordwise-uniform gusts. The load response given by Theodorsen to a chordwise uniformly acting gust is: ${ }^{3}$

$$
C l^{\prime}=\left[2 \pi k i C\left(f_{\text {red }} / 2\right)-\pi\left(f_{\text {red }} / 2\right)^{2}\right] \frac{\alpha}{0.5 * \text { chord }} e^{i \omega t}
$$

The function $C\left(f_{\text {red }} / 2\right)$ is the Theodorsen function, ${ }^{4}$ which uses the reduced frequency to determine both the lift modifier due to the aerodynamic damping caused by the unsteady wake, and the added mass effect. Theodorsen's function is expressed in terms of Hankel functions $(\mathrm{H})$, using the reduced frequency given in equation 1 as an argument:

$$
C\left(f_{\text {red }} / 2\right)=F\left(f_{\text {red }} / 2\right)+i G\left(f_{\text {red }} / 2\right)=\frac{H_{1}^{(2)}\left(f_{\text {red }} / 2\right)}{H_{1}^{(2)}\left(f_{\text {red }} / 2\right)+i H_{0}^{(2)}\left(f_{\text {red }} / 2\right)}
$$

The Hankel function is defined as $H_{v}^{(2)}\left(f_{\text {red }} / 2\right)=J_{v}\left(f_{\text {red }} / 2\right)+i Y_{v}\left(f_{\text {red }} / 2\right)$, where $J_{v}$ and $Y_{v}$ are Bessel functions of the first and second kind, respectively. For sinusoidal gusts, the Sears function, developed by von Karman and Sears, ${ }^{5}$ gives the lift coefficient as: ${ }^{3}$ 


$$
C l^{\prime}=2 \pi\left(\frac{\alpha}{U_{\infty}}\right) S\left(f_{r e d} / 2\right) e^{i 2 \pi U_{\infty} / \omega}
$$

The function $S\left(f_{\text {red }} / 2\right)$ is the Sears function, given by:

$$
S\left(f_{\text {red }} / 2\right)=\left[J_{0}\left(f_{\text {red }} / 2\right)-i J_{1}\left(f_{\text {red }} / 2\right)\right] C\left(f_{\text {red }} / 2\right)+i J_{1}\left(f_{\text {red }} / 2\right)
$$

Here $C\left(f_{\text {red }} / 2\right)$ is the Theodorsen function given in equation 3 . At this point it is important to note that the Sears function as given is derived with the gust as referenced to the centre of the aerofoil. If the gust is referenced to the leading edge, a transform must be applied to the Sears function, giving: ${ }^{3}$

$$
\begin{gathered}
\operatorname{Re}\left(S^{\prime}\right)=\operatorname{Re}(S) \cos \left(f_{\text {red }} / 2\right)+\operatorname{Im}(S) \sin \left(f_{\text {red }} / 2\right) \\
\operatorname{Im}\left(S^{\prime}\right)=-\operatorname{Re}(S) \sin \left(f_{\text {red }} / 2\right)+\operatorname{Im}(S) \cos \left(f_{\text {red }} / 2\right)
\end{gathered}
$$

In an application where the aerofoil is rotating, such as a helicopter or a tidal turbine, the wake forms a helical shape downstream of the aerofoil and therefore comes back into close proximity with the aerofoil $\mathrm{Nb}$ times per revolution (where $\mathrm{Nb}$ is the number of aerofoils on the rotor). This returning wake effect has an impact on the unsteady load response and so must be modelled in rotary applications. There is no correction for returning wake effects associated with the Sears' function, but Loewy ${ }^{22}$ developed a modifier to the Theodorsen function to account for the returning wake, by replacing the Theodorsen function in equation 2 with: ${ }^{3}$

$$
C_{L}\left(f_{\text {red }} / 2, \frac{\omega}{\Omega}, h\right)=\frac{H_{1}^{(2)}\left(f_{\text {red }} / 2\right)+2 J_{1}\left(f_{\text {red }} / 2\right) W}{H_{1}^{(2)}\left(f_{\text {red }} / 2\right)+i H_{0}^{(2)}\left(f_{\text {red }} / 2\right)+2\left(J_{1}\left(f_{\text {red }} / 2\right)+i J_{0}\left(f_{\text {red }} / 2\right)\right) W}
$$

$C_{L}\left(f_{\text {red }} / 2\right)$ is called the Loewy function. The complex value function $W$ accounts for the number of blades and the relative phase of the gust and blade rotation. With $N_{b}$ blades it is given by: ${ }^{3}$

$$
W=\left(e^{f_{r e d} h / 0.5 c} e^{i 2 \pi\left(\omega / N_{b} \Omega\right) e^{i(\Delta \Psi) \omega / \Omega}}-1\right)^{-1}
$$

The parameter $h$ is the spacing between successive wakes, which is assumed to be constant, and $\Delta \Psi$ is the quarter-wavelength of the wake. Note that if $\omega / N_{b} \Omega$ is an integer, then all the shed wake effects are in phase, creating a wake resonance condition which has significant effects on the blade loading. This will be shown in the results section on rotors (section III.E).

This study aims to investigate the effects of 3D geometry on the load response to unsteady gusts, and as such the gusts applied are generally $2 \mathrm{D}$ with respect to local conditions, so that there is no spanwise component of incident flow. The two gust types used are sinusoidal gusts and uniform gusts, given by:

$$
\begin{gathered}
\text { Sinusoidal: } u_{\text {gust }}=\alpha u_{\infty} \exp \left(i \omega\left(t-\frac{x}{u_{\infty}}\right)\right) \\
\text { Uniform: } u_{\text {gust }}=\alpha u_{\infty} \exp (i \omega t)
\end{gathered}
$$

Here $\alpha$ gives the oscillation amplitude, as a proportion of the freestream velocity $u_{\infty}$. The gust is transverse, oscillating orthogonally to the freestream velocity $u_{\infty}$ for the finite-wing geometries, with no spanwise component. In the case of rotating geometries, the uniform gust is used oscillating in the axial flow direction, which in terms of the relative velocity at each blade section generates an approximately transverse uniform gust. The 2D response to sinusoidal gusts is predicted using the Sears function (equation 4), while the uniform gust response is predicted using the Theodorsen function (equation 2), for non-rotating geomteries and the Loewy function (equation 8) for rotating geometries.

Unsteady load response is quantified using the response function, $R$. This is a measure of the unsteady lift coefficient as compared to the $2 \mathrm{D}$ quasi-steady lift response, $2 p i \alpha$. The response function is given by:

$$
R\left(f_{\text {red }}\right)_{2 D}=\frac{L^{\prime}}{\pi \alpha u_{\text {rel }}^{2} A e^{i \omega t}}=\frac{C l^{\prime}}{C l_{\text {quasi-steady }}}
$$

This is effectively the ratio between the unsteady and the steady lift coefficient. Throughout this study comparison between 2D and 3D lift response will be made using the response function, where the magnitude 
$|R|$ denotes the magnitude of the unsteady load compared to quasi-steady response, and the phase $\angle R$ denotes the lag between the unsteady lift response and the forcing function (the gust-induced angle of attack). Occasionally, comparison will also be made to the local 3D quasi-steady lift distribution, and for this an equivalent $3 \mathrm{D}$ response function will be used. This is given by:

$$
R\left(f_{\text {red }}\right)_{3 D}=\frac{C l^{\prime}}{C l_{\text {steady }}}
$$

where $C l_{\text {steady }}$ is obtained from the steady $3 \mathrm{D}$ lift distribution calculated by the vortex lattice model. Using $R\left(f_{r e d}\right)_{3 D}$ is therefore a way to account for the quasi-steady contribution of the wake-induced downwash, which is inherent to $3 \mathrm{D}$ finite-wing geometries.

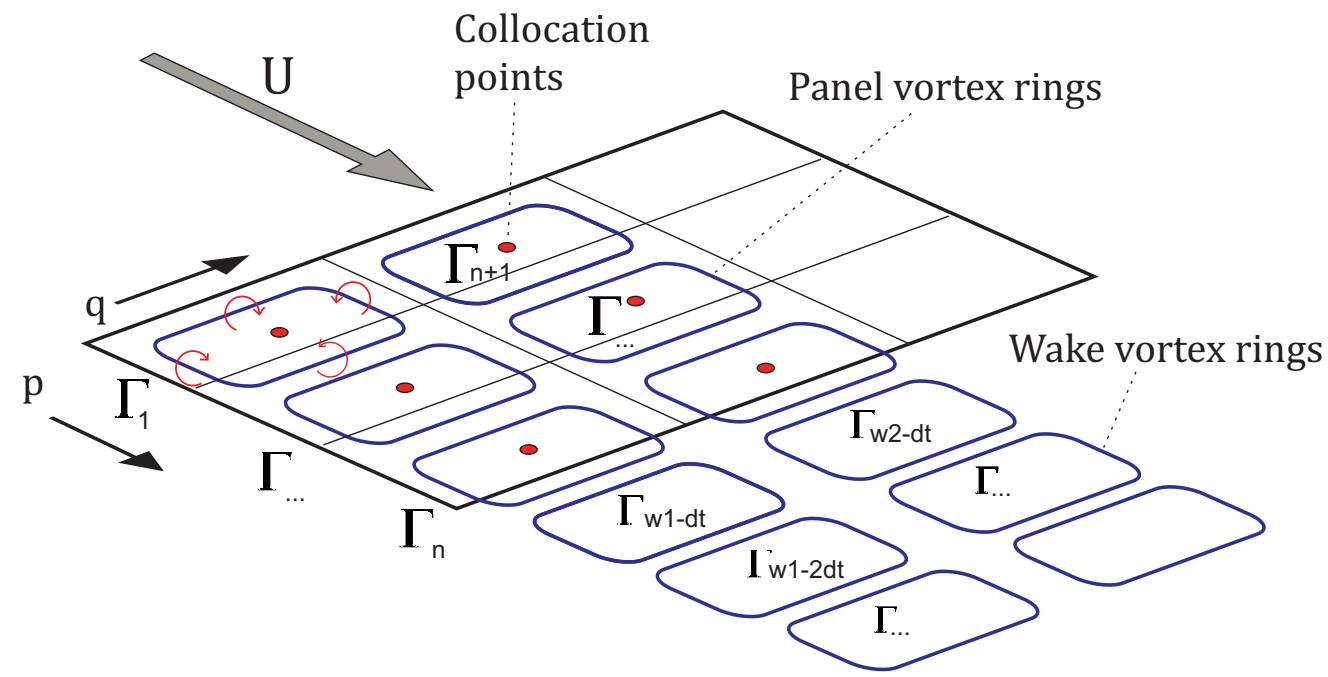

Figure 1. Illustration of the basic structure of the vortex lattice model in $3 D$.

\section{II.B. The unsteady vortex lattice model}

The vortex lattice method used in this study is based on that of Katz and Plotkin, ${ }^{18}$ with modifications to allow for harmonic flow. The model can be used in 2D and 3D, with similar underlying principles. An illustration of the 3D panel model can be seen in Figure 2. The aerofoil is discretised into panels with associated vortex rings providing the aerofoil circulation shown in blue in figure 2. Each panel also has a collocation point at three-quarter chord (red dot), where the boundary conditions are evaluated. This placement of the collocation point implicitly ensures that the Kutta condition is met. Vortex panels are shed into the wake at the steady freestream speed.

In order to find the unknown circulation of each vortex panel and wake, a matrix equation is created based on Neumann's boundary condition that the induced flow normal to the aerofoil surface must sum to zero. This boundary condition is applied to each panel collocation point, and the Biot-Savart law is used to find the influence of each panel and wake vortex on each collocation point. The resulting matrix equation has the following form:

$$
\begin{gathered}
\left(A \Gamma+u_{\infty}+u_{\text {gust }}+u_{\text {wake }}\right) \cdot n=0 \\
(A \Gamma) \cdot n=-\left(u_{\infty}+u_{\text {gust }}+u_{\text {wake }}\right) \cdot n=-u . n
\end{gathered}
$$

The matrix $A$ is the influence matrix, which contains information about the aerofoil geometry and wake shape, and effectively applies the Biot-Savart law to each lattice panel and wake vortex, finding the induced velocity at each collocation point for unit circulation. $\Gamma$ is the vector of unknown panel and wake circulation strengths. The components of the matrices are: 


$$
\left[\begin{array}{cccccccc}
a_{1,1} & a_{1,2} & \ldots & a_{1, n}+b_{1,1} & a_{1, n+1} & a_{1, n+2} & \ldots & a_{1, N}+b_{1, m} \\
a_{2,1} & a_{2,2} & \ldots & a_{1, n}+b_{2,1} & a_{2, n+1} & a_{2, n+2} & \ldots & a_{1, N}+b_{2, m} \\
\vdots & \vdots & \ddots & \vdots & \vdots & \vdots & \ddots & \vdots \\
a_{N, 1} & a_{N, 2} & \ldots & a_{N, n}+b_{N, 1} & a_{N, n+1} & a_{N, n+2} & \ldots & a_{N, N}+b_{N, m}
\end{array}\right]\left[\begin{array}{c}
\Gamma_{1} \\
\Gamma_{2} \\
\vdots \\
\Gamma_{N}
\end{array}\right]=-\left[\begin{array}{c}
u_{1} \cdot n_{1} \\
u_{2} \cdot n_{2} \\
\vdots \\
u_{N} \cdot n_{N}
\end{array}\right]
$$

The influence coefficients $a$ and $b$ apply the Biot-Savart law to the aerofoil panels and wake vortices respectively, to obtain the induced velocity at each collocation point. As this study uses the simplification of frequency-domain calculations, the gusts and resulting circulations vary harmonically in the form:

$$
\Gamma(t)=\Gamma * \exp (i \omega t)
$$

Using this assumption, the circulation strength of the wake can be obtained as a harmonic function of the trailing edge panel circulation, instead of being calculated at each individual time step. This simplifies the analysis and greatly increases the speed of the calculations. As such, for a line vortex starting at point $r_{1}$ and ending at $r_{2}$, acting on a point $r$, the influence coefficients have the following discretised forms for unit circulation:

$$
\begin{gathered}
a_{i, j}=n \cdot\left(K *\left(r_{1} \times r_{2}\right)\right) \\
K=\frac{1}{4 \pi\left|r_{1} \times r_{2}\right|^{2}} *\left(\frac{r \cdot r_{1}}{\left|r_{1}\right|}-\frac{r \cdot r_{2}}{\left|r_{2}\right|}\right) \\
b_{i, q}=\sum_{k_{q}=1}^{\infty} a_{i, k_{q}} e^{-\omega * k_{q} * d t}
\end{gathered}
$$

In this notation, the matrix size is $\mathrm{NxN}$, where $N=n \times m, m$ given by the number of spanwise panels and $\mathrm{n}$ by the number of chordwise panels. Following the notation in Figure $2, i=j=p+m \times(q-1)$, where $i$ refers to the panel at which the induced velocity is being evaluated, and $j$ refers to the panel on the aerofoil or in the wake that is inducing the velocity.

The discretised wake time step $d t$ in equation 20 is determined by consideration to the discretisation of the wake. The wake resolution near the trailing edge has a significant impact on the results, and it was found in this study that the necessary stability and accuracy criterion was that the streamwise wake panel length must be shorter than the aerofoil chordwise panel length. For the results presented here, the distance between wake vortices is always less than or equal to half of the trailing edge panel length. The number of spanwise and chordwise panels used for the aerofoil was increased in each case until the results did not change more than $5 \%$ as the resolution was doubled.

Having calculated the distribution of panel circulation $\Gamma$, the force on each panel is calculated from the cross product of the panel vortex with the local induced velocity, the vector form of the Kutta-Joukowski theorem:

$$
F_{p, q}=\rho *\left(U \times \Gamma_{j}\right)=\rho *\left(\left(u_{r e l}+u_{\text {gust }}+u_{\text {wake }}\right) \times \Gamma_{j}\right)
$$

If the panel evaluated is not at the leading edge, $\Gamma_{j}$ is replaced by $\left(\Gamma_{j}-\Gamma_{j-1}\right)$. The expression $u_{r e l}$ is the local relative steady velocity, which is given by:

$$
u_{r e l}=u_{\infty}+u_{r o t}=u_{\infty}+T S R *\left|u_{\infty}\right| e_{\theta}
$$

TSR denotes the tip-speed ratio, a design parameter denoting the ratio of blade speed to axial freestream speed. Throughout this study, the lift coefficient $C_{L}$ is generally defined with respect to the local flow properties, as this enables comparison to equivalent $2 \mathrm{D}$ conditions. As such, the lift coefficient is given by: 


$$
C_{L}=\frac{L}{0.5 \rho u_{r e l}^{2} A_{q}}
$$

$A_{q}$ refers to the total area of a strip of panel elements, at given spanwise position as indicated by $q . L$ is obtained from equation 21.

Blade thickness effects are not modelled, and the effect of the turbine on the upstream flow is not considered; the gusts are assumed to convect unchanged towards the turbine (the frozen gust assumption).

a)

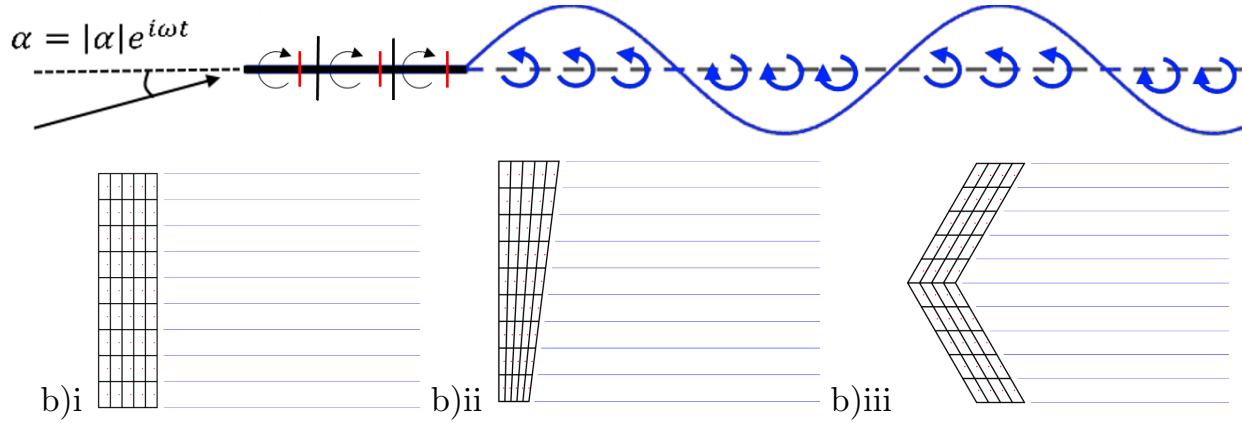

$$
\text { c)i }
$$

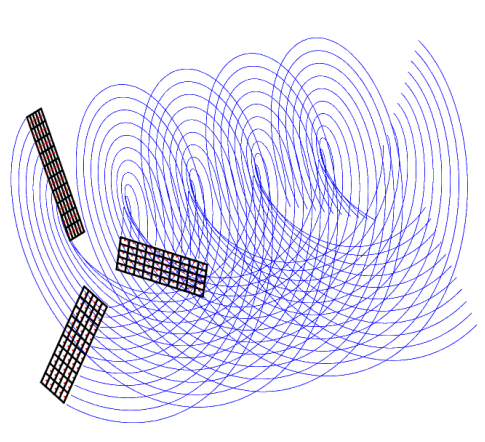

c)ii

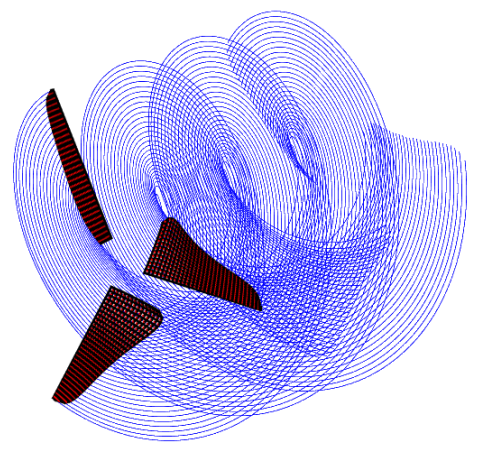

Figure 2. Illustration of the different geometries considered, using the vortex lattice method. Black lines represent the aerofoil geometries, and blue lines represent the path of the wake vortices.

\section{Results}

Figure 2 shows the selection of geometries used in the present paper. Figure 1a) represents the simple 2D flat plate model which is used to derive the transfer functions from classical unsteady aerofoil theory. This 2D geometry is used to validate the unsteady panel method, showing that it can resolve the unsteady flow to the same accuracy as the transfer functions from classical aerofoil theory. Figures $2 \mathrm{~b}$ )i-b)iii show a series of finite wing geometries. The spanwise geometry parameters investigated are aspect ratio, taper and sweep. The blade sections are flat plates at zero incidence, similar to the 2D model in Figure 2a). Finally, two rotor geometries are shown in figure 2c). Figure 2c)i shows a rotor geometry, of an untwisted blade with flat plate aerofoil sections. The blade is aligned so that the steady angle of attack at the tip is zero. Figure 2c)ii shows a turbine with realistic camber, chord and twist distribution, based on a model tidal turbine which has been tested in steady and unsteady conditions. ${ }^{23}$ This turbine design gives an indication of the combined effect of various 3D geometry features on the unsteady response of a real turbine.

\section{III.A. Validation}

The steady response of the 3D geometries has been compared with the baseline cases available in Katz and Plotkin, ${ }^{18}$ which have themselves been verified against experimental data for tapered, swept and rotating blades. A summary of the validation results can be seen in Figure 3. Figure 3(a) shows the spanwise lift variation of a wing with aspect ratio 4, at zero sweep, 45 degrees sweep, and 135 degrees sweep. Figure 3(b) shows the spanwise lift variation for a wing with aspect ratio 7.28 and with different taper ratios. Figure $3(\mathrm{c})$ shows the total lift coefficient of aerofoils with varying aspect ratio, at zero and 45 degrees sweep. The 
vortex lattice model generally agrees well with these baseline cases, apart from at the limit of infinite taper ratio $(\lambda=0.0$, green line in Fig. 3(b)). This is an extreme case, which will not be considered in the study. As such, the tapered wing results are satisfactory.
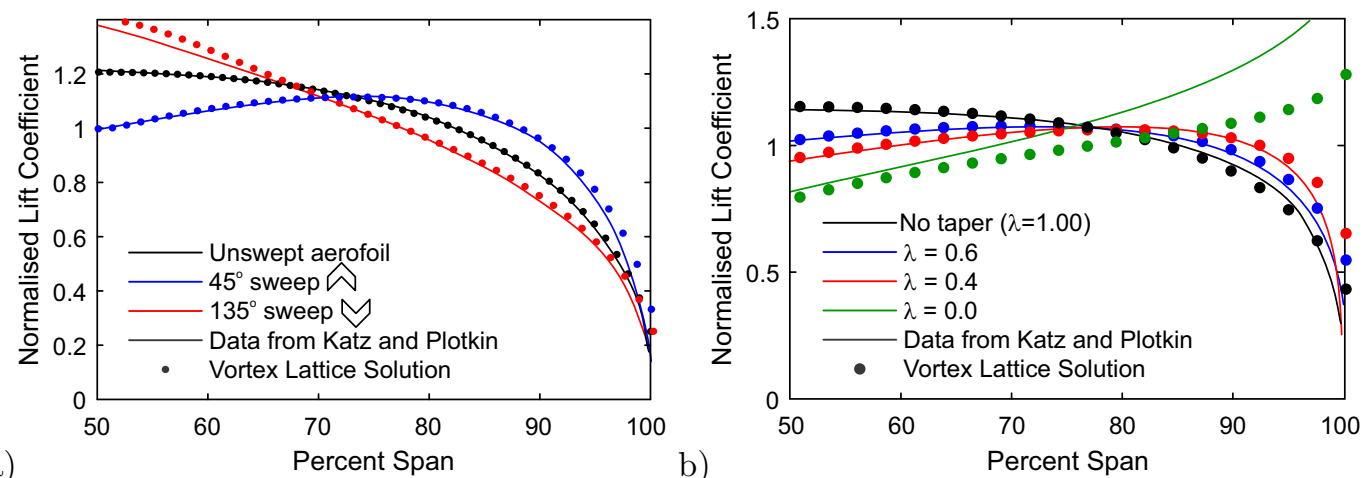

a)
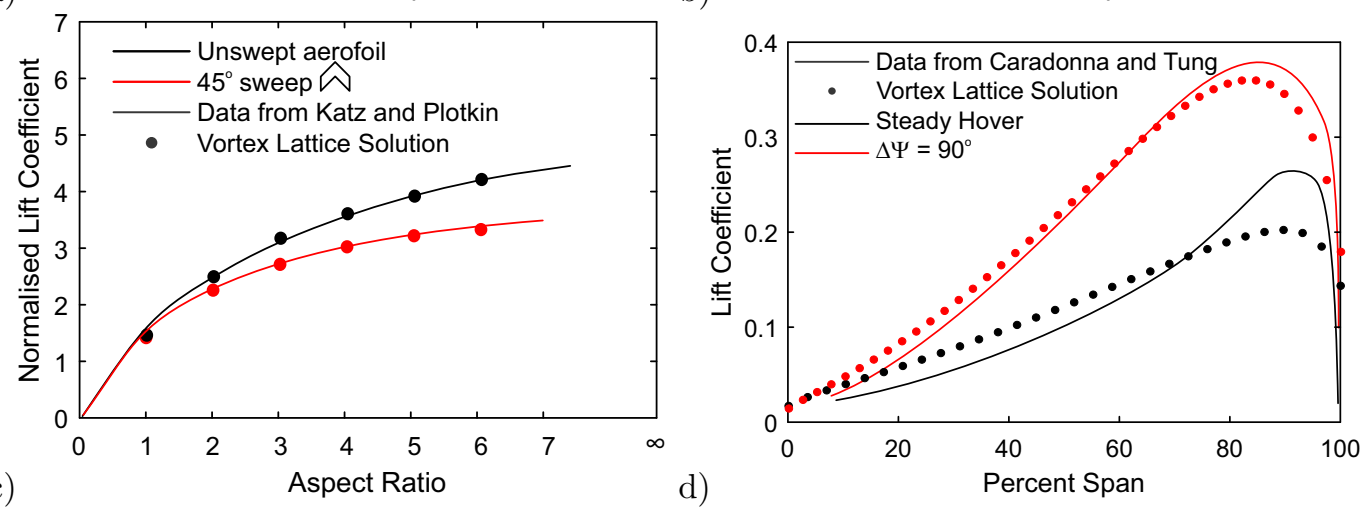

Figure 3. validation of steady vortex lattice model results against baseline cases from Katz and Plotkin. ${ }^{18}$

Figure 3(d) shows a comparison of the vortex lattice results with those obtained by Katz and Plotkin's ${ }^{18}$ time-domain vortex lattice code, which was validated against an experimental study by Caradonna and Tung. ${ }^{24}$ This case has been used for validation of panel codes in other studies (see for example Tan and Wang ${ }^{25}$ ). The aerofoil is a NACA 0012, which in this study is modelled as a flat plate. The two states considered are the flow state after a quarter-rotation of the blades (red line), and a "steady-hover" condition in which the wake is allowed to develop and remains close to the rotor (black line).

While a lack of available geometry information means that it is difficult to replicate the conditions in the study exactly, the results agree reasonably well for the case of wake propagation to a quarter rotor revolution. The agreement of the vortex lattice solution with the steady hover results of Katz and Plotkin is, however, not as good. This discrepancy may be explained by the effect of vortex rollup, which is present in the experiments and is modelled by the time-domain solver. In the case of a helicopter rotor configuration, wake rollup causes the wake to move inwards towards the axis of rotation, and therefore results in increased wake influence in the mid-span region, reducing the loading in this region. In the harmonic vortex lattice method used here, wake rollup is not modelled, and so a more linear variation in lift coefficient along the span is predicted. In the case of a tidal turbine, the effect of wake rollup is to move the wake away from the axis of rotation, and therefore concentrate any wake effects to the blade tip region. However, unlike a helicopter rotor in "steady hover" conditions, the wake of a tidal turbine convects downstream with the bulk flow, and therefore the wake rollup will be minimal while the wake is near the turbine. Nevertheless, quantifying the effect of wake rollup on the unsteady response will be considered for a future study.

Figure 4 shows the magnitude (Fig. 4(a)) and phase (Fig 4(b)) of the load response of a 2D aerofoil to a sinusoidal gust, as calculated by the 2D vortex lattice method for the flat plate shown in Fig. 2(a). For comparison, Sears' function is shown. It can be seen that the agreement between the model and classical aerodynamic theory is good, showing that the vortex lattice captures the relevant inviscid unsteady flow 
physics.

The validation cases given above show that the vortex lattice model captures the correct 3D steady distribution of lift and the correct $2 \mathrm{D}$ unsteady effects. This gives confidence that the $3 \mathrm{D}$ unsteady results discussed below are reliable and can be used to elucidate the flow physics governing 3D unsteady load response.
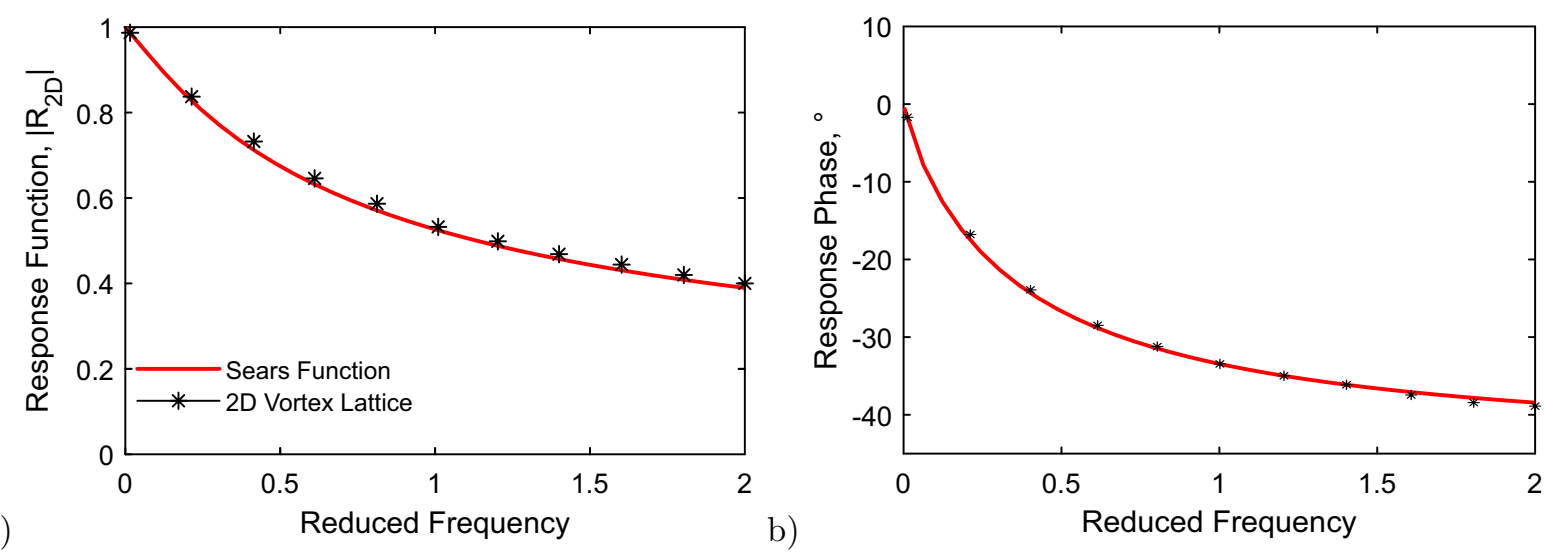

Figure 4. validation of the 2D unsteady vortex lattice model against classical aerofoil theory.

\section{III.B. Finite wing}

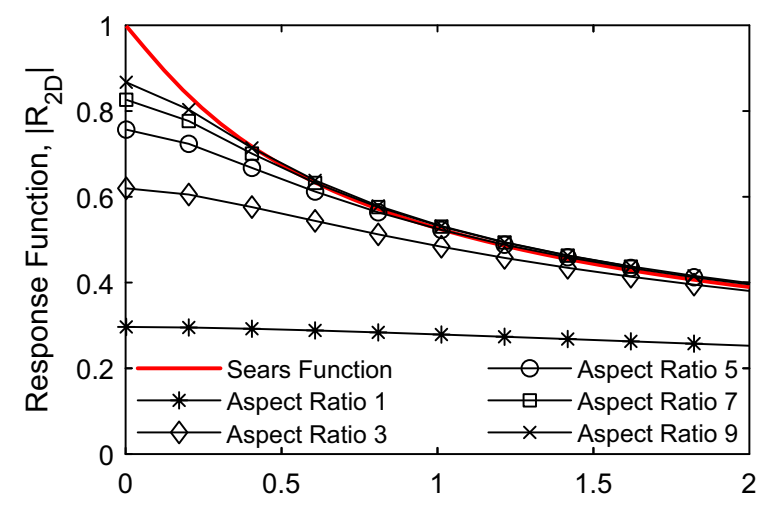

a)

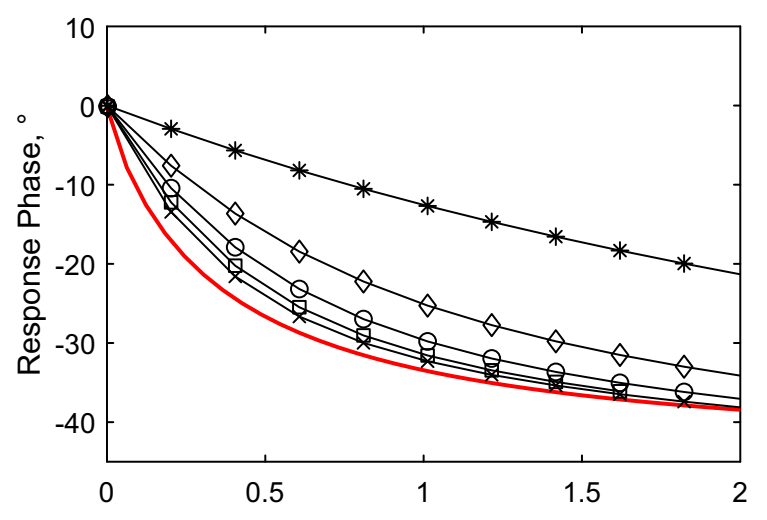

b)

Reduced Frequency

Figure 5. Mid-span unsteady response of finite wings with different aspect ratios, interacting with a sinusoidal gust. The response function is a measure of the unsteady load relative to the quasi-steady load, and is given by equation 12 .

In this section, the effect of aspect ratio on the unsteady load response is investigated. Finite wings with flat plate aerofoil cross sections are considered at different aspect ratios. Sinusoidal gusts, as given in equation 10, and uniform gusts as given by equation 11 are applied and compared with the 2D Sears and Theodorsen functions, respectively. There is no spanwise variation in gust shape or amplitude.

Figures 5 and 6 show the mid-span lift response of aerofoils with a range of aspect ratios, showing both the amplitude and phase of the load response, given by the magnitude and phase angle of the response function (equation 12). Figure 5 show the response to a sinusoidal gust, compared to prediction by the Sears function, while Figure 6 shows the response to a uniform gust, compared to the Theodorsen function. As described above, the load has been non-dimensionalised by the quasi-steady 2D load. The discrepancy between the 2D characteristic and the vortex lattice at zero reduced frequency (quasi-steady response) is due to the inherent reduction in steady lift in a finite wing (see Figure 3(c)). However, as the reduced frequency increases the mid-span load response of a finite wing can be seen to approach the $2 \mathrm{D}$ response in both the sinusoidal (Figure 5) and uniform (Figure 6) gust cases. The rate at which this approach happens increases with the aspect ratio of the aerofoil, with the aspect ratio 1 aerofoil showing a substantially lower response 


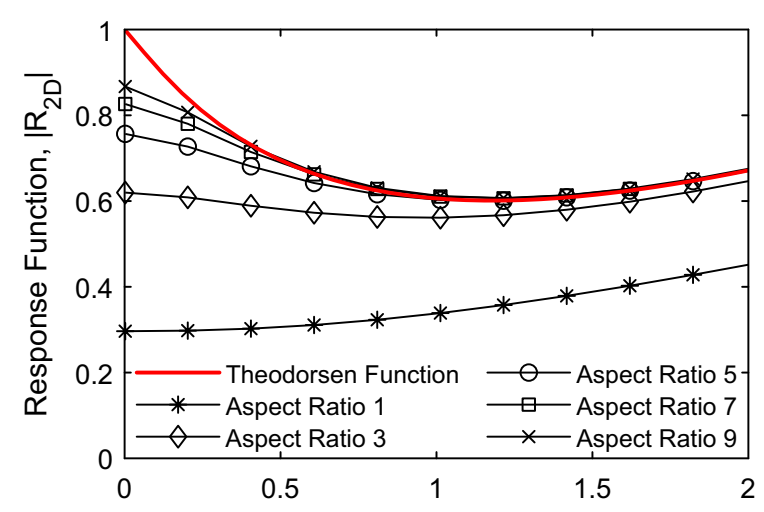

a) Reduced Frequency

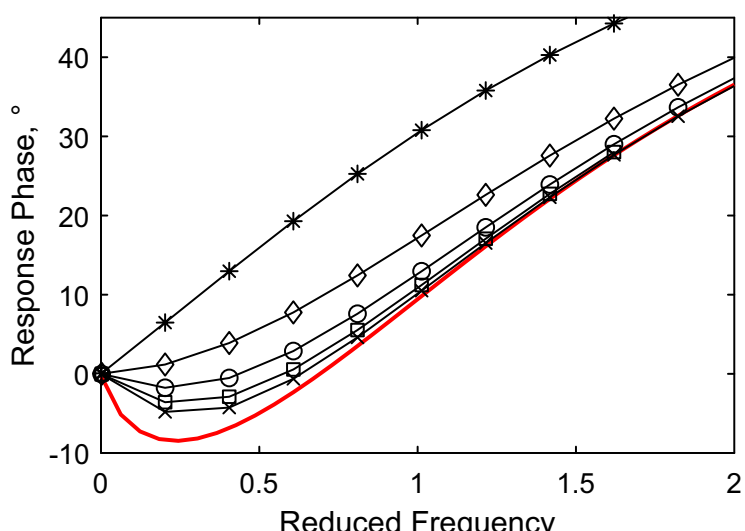

b)

Figure 6. Mid-span unsteady response of finite wings with different aspect ratios, interacting with a uniform gust. The response function is a measure of the unsteady load relative to the quasi-steady load, and is given by equation 12 .

even at large reduced frequency. On the other hand, the phase of the response (Figures 5(b) and 6(b)) does not approach the 2D prediction as rapidly, and only reaches the $2 \mathrm{D}$ curve at large reduced frequency and aspect ratios $\left(f_{\text {red }}>1\right.$ and $\left.\mathrm{AR}>7\right)$.

As well as a variation in mid-span lift response, the loading on a finite wing will vary across the span. This is shown in Figure 7, which gives the spanwise distribution of response for different aspect ratios at a reduced frequency of 1.0. It can be seen that, for all reduced frequencies, the unsteady load response reduces as the wing tip is approached. As the aspect ratio increases, a larger proportion of the span exhibits 2D behaviour, although the lift magnitude (Figure 7(a)) approaches 2D predictions faster than the phase (Figure 7(b)) - with an aspect ratio of 9, the lift magnitude is equal to the $2 \mathrm{D}$ case over the middle $60 \%$ of span, whereas the phase only approaches the $2 \mathrm{D}$ phase over the middle $20 \%$. The phase variation in figure 7 (b) also shows that at any given aspect ratio and frequency, the tip response leads the mid-span response. So the tip and the mid-span do not respond in phase with each other, even when there is no spanwise gust variation. The results in figure 7 are obtained for a uniform gust, but they are very similar for a sinusoidal gust, suggesting the same underlying mechanism.
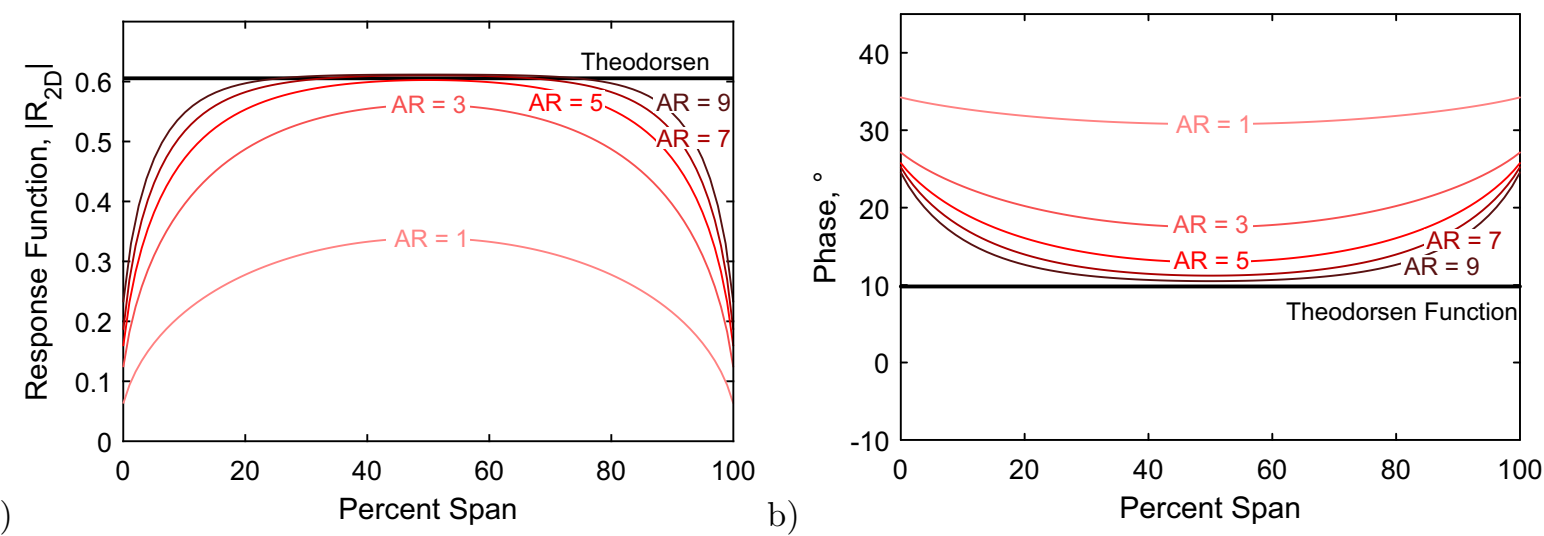

Figure 7. spanwise distribution of unsteady load amplitude (a) and phase (b), showing how the distributions change with aspect ratio at a fixed reduced frequency 1.0.

As was noted in figures 5 and 6 , the lift response approaches the $2 \mathrm{D}$ characteristic with increasing reduced frequency. Figure 8 shows the spanwise variation of lift amplitude and phase for an aerofoil with aspect ratio 5 at different reduced frequencies. In the case of the load amplitude (Figure 8(a)), the mid-span response is again closer to the 2D load than that of the wing tip, where the amplitude hardly changes with reduced frequency. This means that the difference in load response between the tip and mid-span increases with reduced frequency. This is seen more strongly in the phase plot (Figure $8(\mathrm{~b})$ ), where the phase response near the tip actually moves further away from the $2 \mathrm{D}$ prediction with increasing reduced frequency. This 
change in phase across the span has significant implications for parameters such as root bending moment, where the integral of the load along the wing is important, as the contributions at each spanwise location will be out of phase with one another.
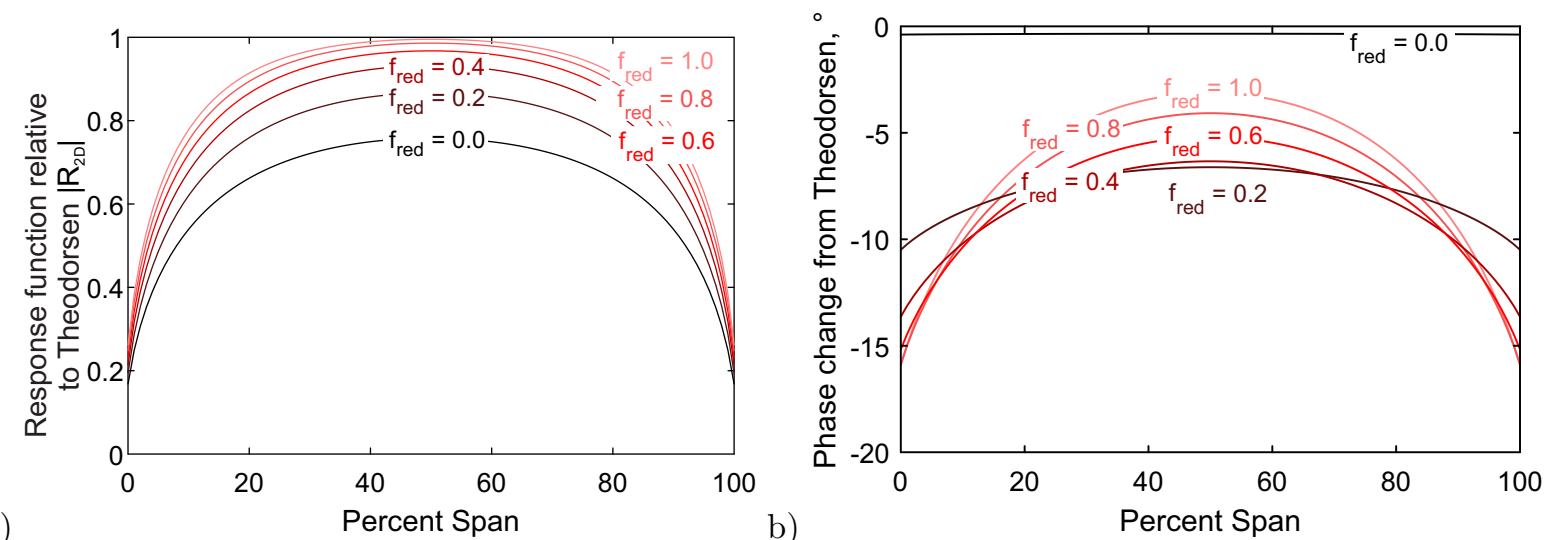

Figure 8. spanwise distribution of unsteady load amplitude (a) and phase (b), showing how the distributions change with reduced frequency at fixed aspect ratio 5.

The findings in Figures 7 and 8 emphasise that the difference between tip and mid-span response increases with reduced frequency in terms of both magnitude and phase. It is important to note that this difference, and the unsteady response characteristic at various spanwise positions, is not proportional to the steady $3 \mathrm{D}$ lift distribution (effectively shown by the $f_{r e d}=0.0$ line in figure $8(\mathrm{a})$ ). The lift characteristics at different spanwise positions cannot be accounted for simply by considering the steady 3D load variation induced by the tip vortices the unsteady flow also causes a significant change to the tip vortices.

The reason for the differences in response between 2D and finite wings can be found by examining the spanwise distribution of wake-induced downwash. The downwash acts to counteract the unsteady change in incidence, and thus provides damping to the unsteady response. Figure 9 shows the downwash at reduced frequencies 0.0 and 1.0 for a wing with aspect ratio 5 (Figure 9(a)) and one with aspect ratio 10 (Figure $9(\mathrm{~b})$ ), both interacting with a sinusoidal gust. The wake influence has been divided into two parts: the downwash created by the spanwise wake component, and that created by the streamwise wake component. In $2 \mathrm{D}$ unsteady analysis, only the spanwise wake is accounted for. In $3 \mathrm{D}$ quasi-steady analysis, only the streamwise downwash at reduced frequency 0.0 is accounted for. Observing the change in these two downwash components with reduced frequency explains the behaviour of 3D unsteady load response.

First, the downwash induced by the spanwise wake (thin black line in figure 9) decreases when approaching the wing tips; this is due to the wake being finite. This is a deviation from $2 \mathrm{D}$ analysis, which assumes an infinite spanwise wake, and is the first source of the 3D unsteady behaviour observed above. The second effect is that of the unsteady streamwise wake downwash. The downwash induced by the streamwise wake starts out equivalent to the quasi-steady 3D downwash inherent to finite wings (red dashed line in figure 9). As the reduced frequency is increased (black dashed line), the streamwise component of downwash diminishes substantially at midspan. This behaviour has been explained by Namba, ${ }^{10}$ who considered a streamwise vortex line with harmonically varying circulation and showed that the induced downwash by such a vortex line decreases with increasing frequency. In Figure 9 it can also be seen that the downwash from the streamwise wake remains large near the wing tips.

The combination of the streamwise and spanwise downwash for $f_{\text {red }}=1.0$ is shown by the thick black lines in Figure 9. (Note that the two components of downwash are not in phase with one another, and so the amplitudes shown in Figure 9 do not sum exactly to the total.) In both aspect ratio cases, the total downwash is lower at midspan than at the wing tips, meaning that there is less damping of the unsteady response at the midspan than at the tips. This explains why the blade tips exhibit a lower amplitude response than the midspan in Figures 7 and 8. It can also be noted from Figure 9 that, while the total downwash is very similar for the two different aspect ratios, the balance of streamwise and spanwise 


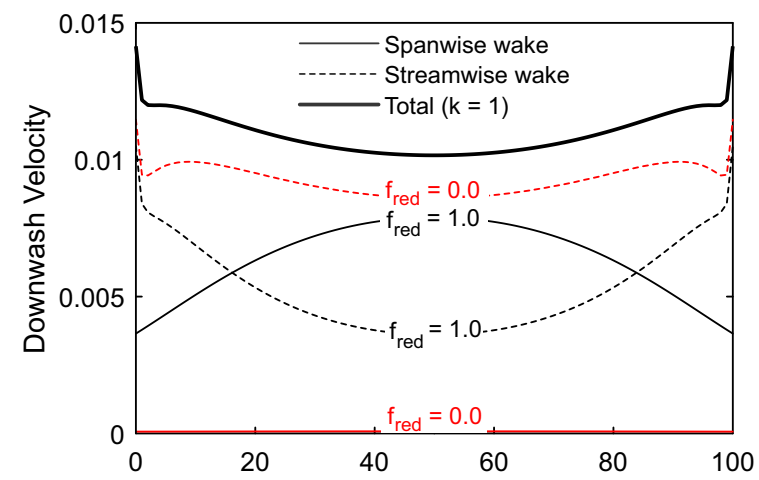

a)

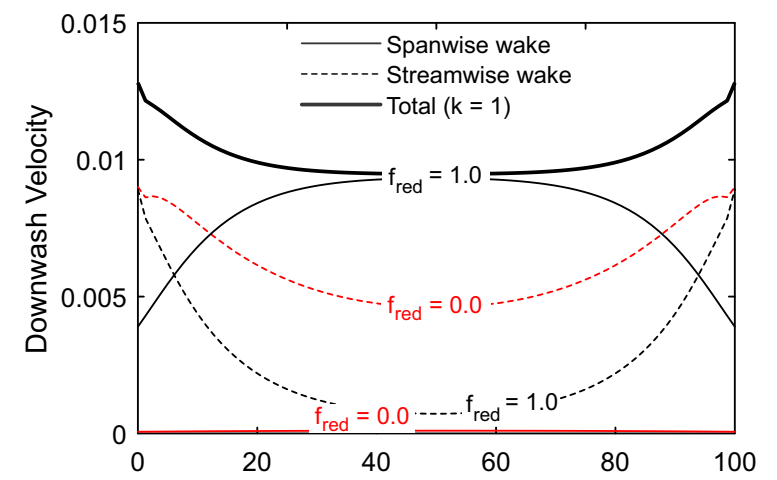

b)

Percent Span

Figure 9. Spanwise distribution of the magnitude of wake-induced downwash, for wings with aspect ratios of 5 (a) and 10 (b). The downwash magnitude is normalised by the steady freestream velocity. The gust is sinusoidal.

components changes with aspect ratio. In figure 9a) (aspect ratio 5), the total downwash is made up of significant streamwise and spanwise wake components, while in Figure 9b) (aspect ratio 10), the downwash at mid-span is almost entirely caused by the spanwise wake. Note also that figure 5(a) shows both of these wings to have $2 \mathrm{D}$ behaviour at mid-span for reduced frequency 1.0, in terms of the load amplitude. We can conclude that for the wing with aspect ratio 5 , the largely $2 \mathrm{D}$ response at mid-span at $f_{\text {red }}=1.0$ is created through a combination of spanwise and streamwise wake downwash effects, both of which are significantly $3 \mathrm{D}$.

Again, the behaviour of the downwash in the case of a uniform and a sinusoidal gust are qualitatively similar, the changes to the load response being caused by the same changes to the wake. From the findings in this sections, we can conclude that for the purpose of modelling a finite wing, the two effects that must be considered are the change to the spanwise wake, and the presence and changing nature of the streamwise wake. Since both components combine to determine the 3D unsteady response, they must both be modelled to get a reliable load estimate.

Furthermore, the important factors for determining the extent of the $3 \mathrm{D}$ flow effects are aspect ratio, the range of reduced frequency considered, and the proximity of the section in question to the wing tips. While long sections of wings at high aspect ratios may exhibit $2 \mathrm{D}$ behaviour in terms of the load magnitude, the phase of the response changes significantly along the span, especially near the tips. This is of particular importance for bending moment estimates, for example, and other analysis where integration of the load along the span is necessary.

\section{III.C. Tapered wing}

Having determined the major effects on aerofoil loading from including the spanwise and streamwise downwash components, we now study the effects of different geometry features, starting with blade tapering. An illustration of a tapered geometry can be seen in figure 2b)ii. Note that as the incident velocity is the same at all spanwise positions, the local reduced frequency (given by equation 1) decreases linearly along the span as the chord length decreases. We consider a wing with aspect ratio 5 (referenced to the mid-span), and with a taper ratio of 0.4 . (Note that this combination of taper and aspect ratio results in an aspect ratio of 3.5 when referenced to the longest chord.)

Figure 10 shows the lift response at the mid span, 5\% span (longer chord) and 95\% span (short chord), against the local reduced frequency. It would appear that response amplitude and phase depends on spanwise position, with the hub, tip and mid span having different response trajectories. However, it can be shown that this apparent change is proportional to the steady lift distribution (Figure 3(b)), and that the governing frequency of the system is the mid-span frequency. Figure 11 shows the same data as figure 10, but non-dimensionalised differently . The load magnitude is expressed as the 3D response function $R_{3 D}$ (i.e. the unsteady load normalised by the local quasi-steady lift coefficient, see equation 13), and is plotted 
against mid-span reduced frequency. For comparison, results are also shown for an untapered wing at aspect ratio 5 (also normalised by the local quasi-steady lift coefficient). The two cases are in very close agreement. This result shows that the mid-span blade properties govern the response of a tapered blade, and that, when non-dimensionalised correctly, the effect of taper is negligible compared with the effect of the wing being finite.
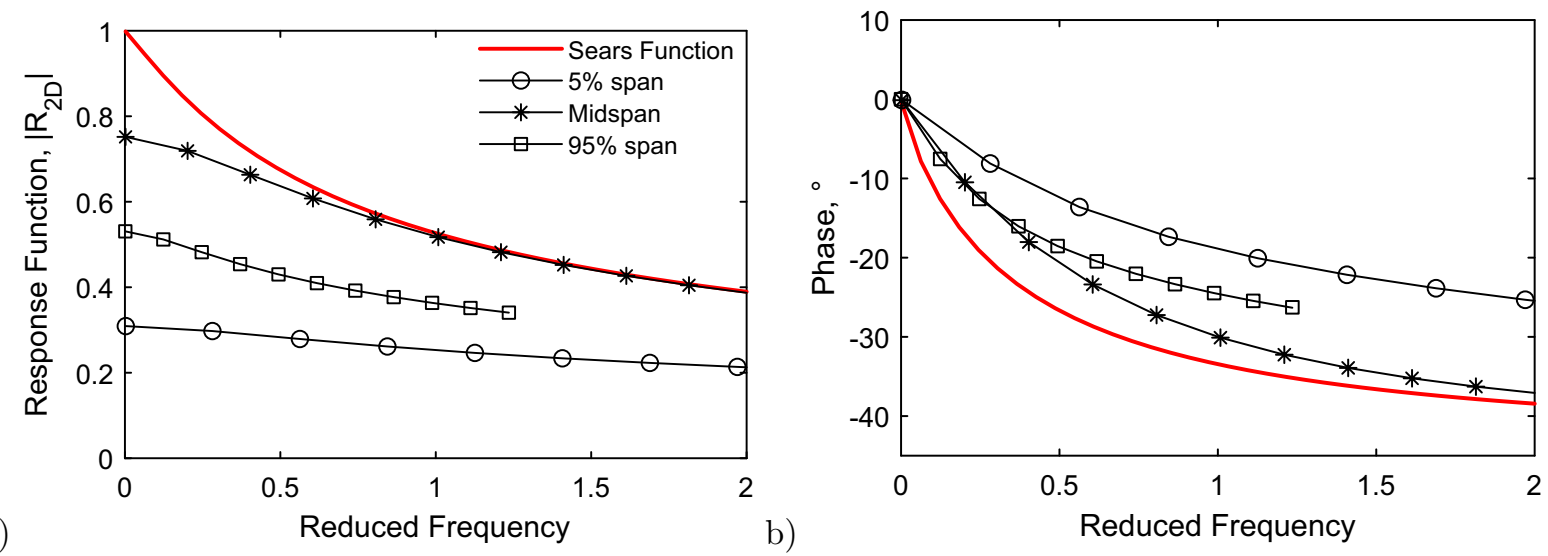

Figure 10. Load response magnitude (a) and phase (b) of a tapered wing with a taper ratio of 0.4 and a midspan aspect ratio of 5 . The load response is given by the response function, defined in equation 12 . THe gust is sinusoidal.
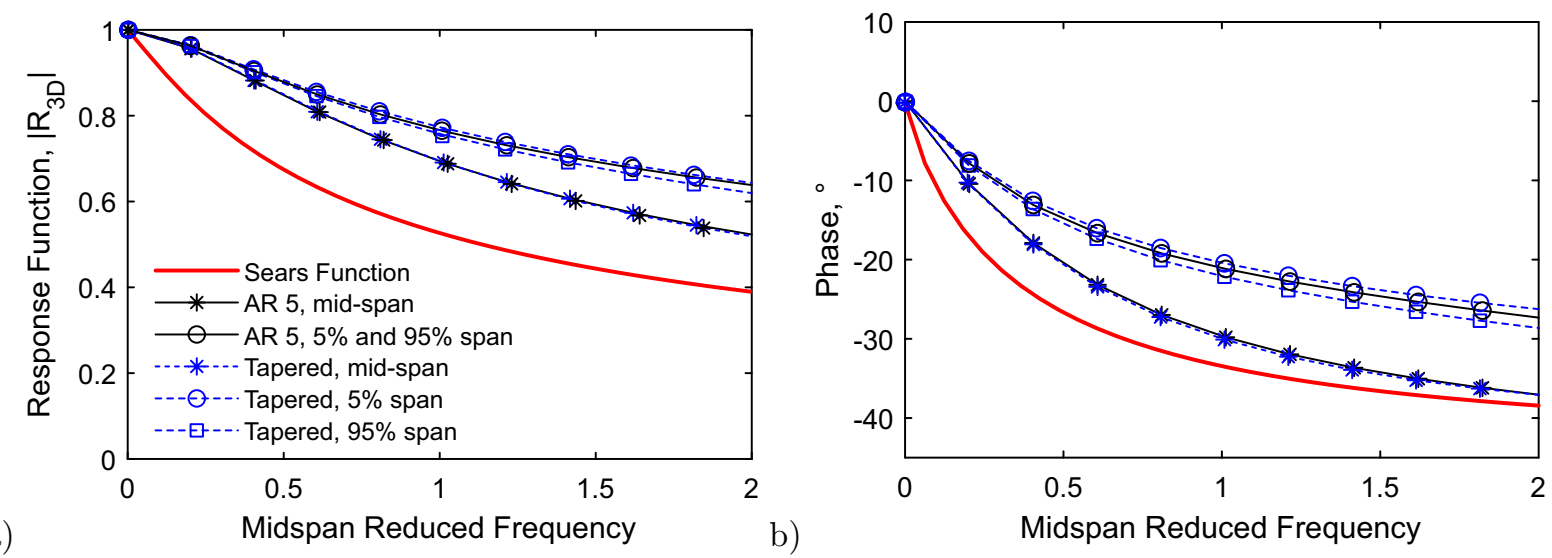

Figure 11. Load response magnitude (a) and phase (b) of a tapered wing with a taper ratio of 0.4 and a midspan aspect ratio of 5 , compared with an untapered blade with an aspect ratio of 5 . The response has been normalised by the $3 D$ steady response, as given by equation 13 . The gust is sinusoidal.

As with the untapered wings considered in section III.B, the unsteady response of a tapered blade is governed by changes in downwash. Figure 12 shows the downwash distribution for the tapered blade, at mid-span reduced frequencies of 0.0 (red lines) and 1.0 (black lines), again divided into the influence of the spanwise and streamwise wakes. Both components of downwash on their own can be seen to vary significantly along the span. The quasi-steady downwash (red dashed line) has a nearly linear spanwise variation; this is caused by the blade taper. By comparison, the total downwash at mid-span reduced frequency 1.0 (thick black line) is more level, due to the combined contribution of the streamwise wake (dashed line) which increases with span and spanwise wake (solid line) which reduces with span. This creates an almost symmetrical response across the span, as seen in Figure 11.

This is evidence of the equalising $3 \mathrm{D}$ effect observed by Namba. ${ }^{10}$ As isolated, $2 \mathrm{D}$ aerofoils, the sections would all follow the same response curve with respect to their local reduced frequency. It can be seen from Figures 10 and 11, however, that this is not the case. When the sections are part of a finite, tapered aerofoil, they all follow the same curve with respect to the midspan reduced frequency, i.e. the interactions between aerofoil sections cause an equalising effect and the response at all spanwise locations tends towards the midspan response. 


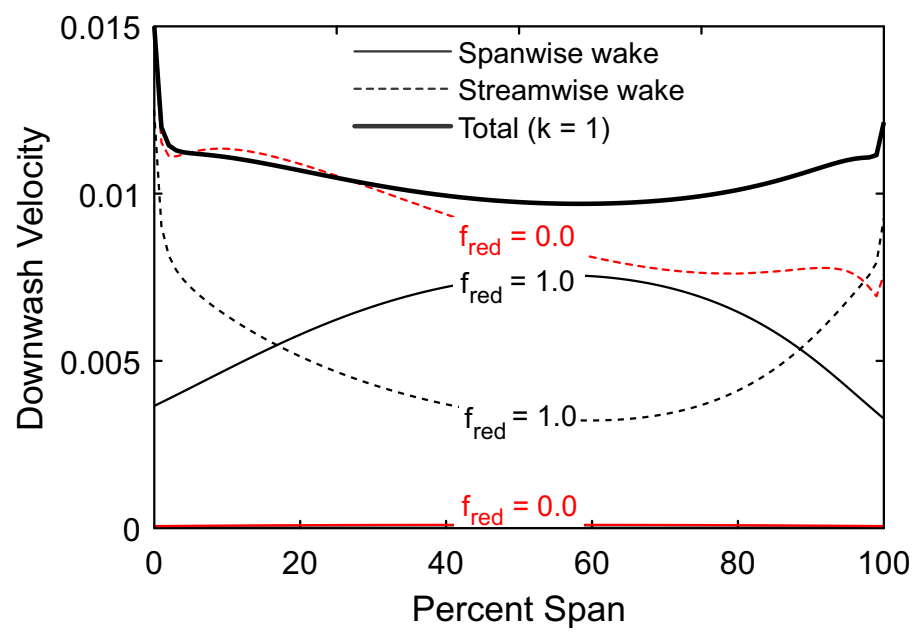

Figure 12. Spanwise distribution of downwash caused by the spanwise and streamwise components of the unsteady wake, for a tapered blade with a taper ratio of 0.4 , and a midspan aspect ratio of 5 . The reduced frequency is referenced to the mid-span. The downwash is normalised by the steady freestream velocity. The gust is sinusoidal.

\section{III.D. Swept wing}

Looking next at the effect of swept wing geometry, a uniform gust interacting with both backward- and forward-swept geometries is considered. A uniform gust is used since its properties do not change in the spanwise direction in the case of a swept blade. (The sinusoidal gust, defined in equation 10, would cause a spanwise phase change in gust properties relative to the leading edge. While this is also an important 3D effect, it is out of scope for the current study.) An illustration of the wing geometry can be seen in figure 2(b)iii, and the effect of sweep on the steady load distribution is shown in Figure 3(a).
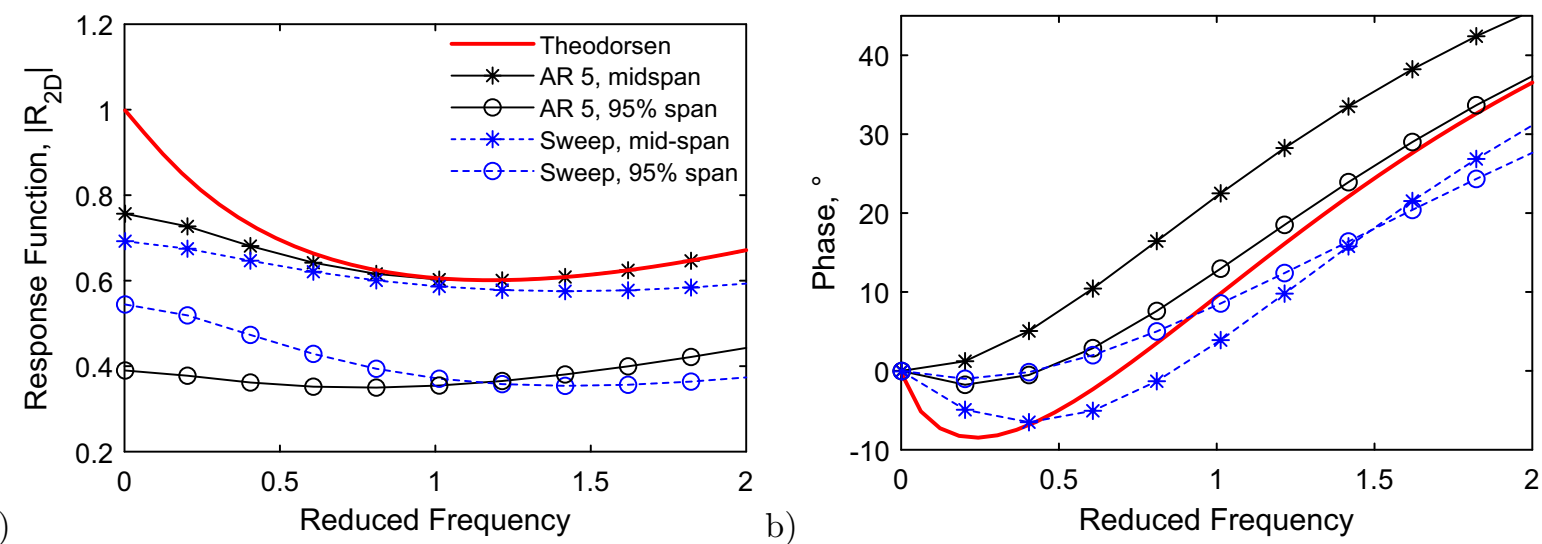

a) Reduced Frequency b)

Figure 13. Load response magnitude(a) and phase (b) of a swept wing with 45 degrees backward-sweep and an aspect ratio of 5 , interacting with a uniform gust.

Figure 13 shows the unsteady lift response for a 45-degree backward-swept wing, with aspect ratio 5. The load magnitude (Fig 13(a)) is affected most dramatically near the wing tip, but there are also significant changes to the phase of the tip and mid-span response, compared to the $2 \mathrm{D}$ and unswept 3D characteristics. Notably, the tip and mid-span response lag behind the 2D prediction for a significant part of the frequency range, and are nearly in phase for the higher reduced frequencies. The mid-span load amplitude is relatively close to the 2D characteristic, and to that of the unswept blade, although it deviates slightly as the reduced frequency increases. The tip load amplitude (at 95\% span) is fairly similar to that of the equivalent position on an unswept blade. These effects are not proportional to the steady lift distribution, unlike the tapered blade case.

Comparing to the response of a 45 degrees forward-swept blade (figure 14), the unsteady effects at mid-span deviate much more notably from the $2 \mathrm{D}$ predictions. In contrast to the results from the unswept 

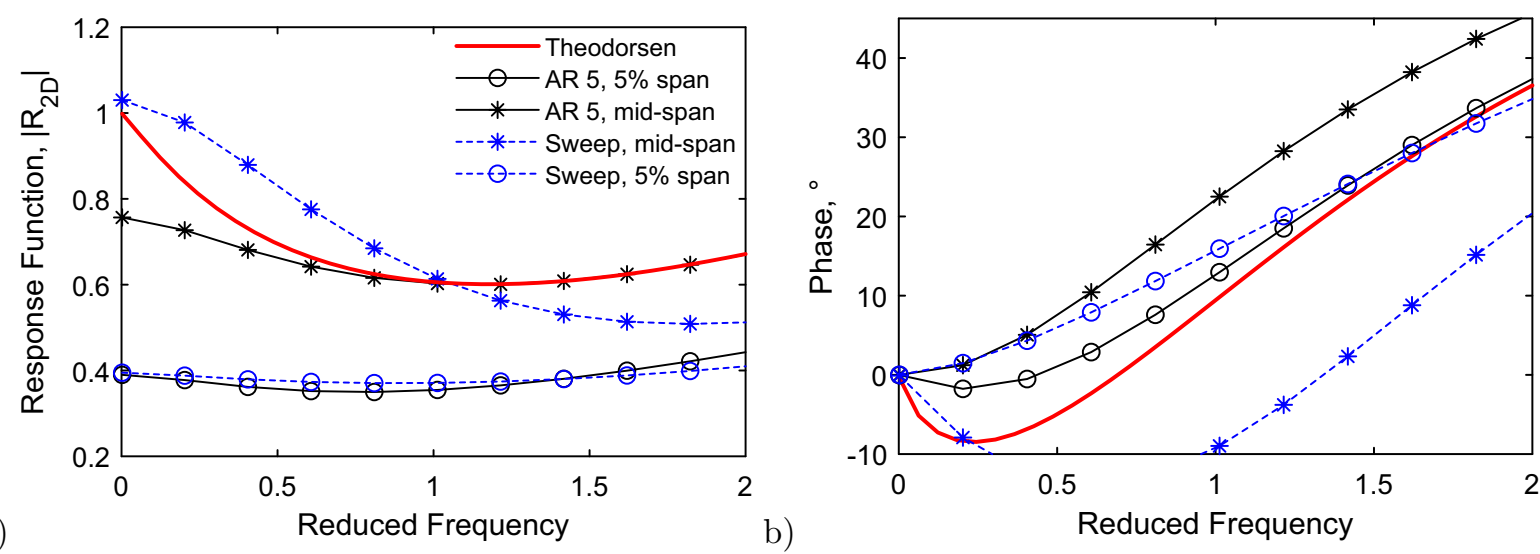

Figure 14. Load response magnitude (a) and phase (b) of a swept wing with 45 degrees forward-sweep and an aspect ratio of 5, interacting with a uniform gust.

geometries, the load amplitude is actually under-predicted by the $2 \mathrm{D}$ characteristic for the reduced frequency range 0.0 to 1.0. Looking at the phase of the response (14(b)), it is the phase at the wing tip that corresponds more closely to $2 \mathrm{D}$ predictions at higher frequencies, while the mid-span phase lags significantly behind $2 \mathrm{D}$ predictions. This result shows the opposite trend compared to earlier results.

Again, it is notable that the change in unsteady response characteristic is not proportional to the steady lift distribution, but is a function of the change of both streamwise and spanwise vorticity due to 3D effects. As opposed to the tapered blade, the response characteristic cannot be reconciled with that of an unswept blade through correct non-dimensional properties.
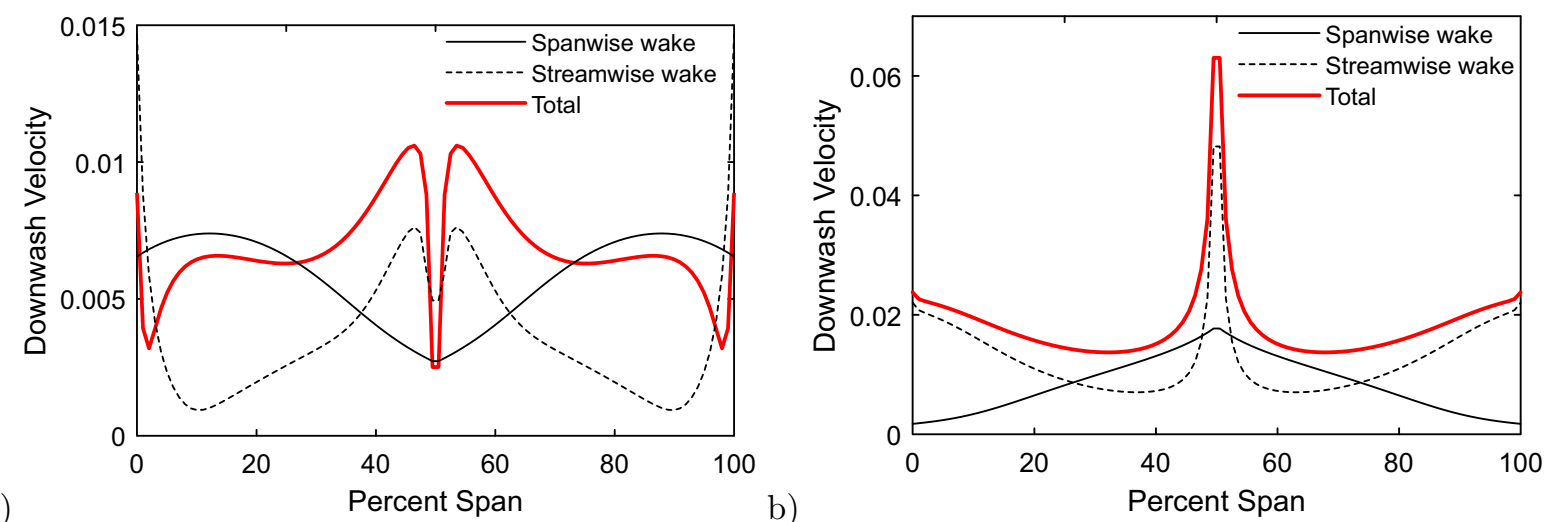

a)

Percent Span

b)

Figure 15. Spanwise downwash distribution of swept wing with 45 degrees backward-sweep (a) and 45 degrees forwardsweep (b), at aspect ratio 5. The reduced frequency is 1.0. The downwash is normalised by the steady freestream velocity. Both cases are interacting with uniform gusts (eq. 11).

The distribution of wake downwash for the backward-swept and forward-swept blades are shown in figure 15, for reduced frequency 1.0. The total induced downwash can be seen to be strongly dependent on both the streamwise and the spanwise components of the wake. As opposed to the finite and tapered wings tested earlier, this is an example of a geometry where the two wake components do not combine to produce a largely $2 \mathrm{D}$ response. In the outboard sections of the backward-swept wing (15(a)), the spanwise wake influence contributes the most to the total downwash. Near the mid-span on the other hand, the streamwise wake effect is strongly felt. This leads to a much more complex unsteady flow behaviour than cannot be captured by 2D models. Considering the forward-swept wing (15(b)), the total downwash exhibits the influence of both wake components throughout most of the span, and the mid-span downwash is much larger than any value seen in other cases (the y-axis has been extended to 4.5 times larger than other downwash plots to accommodate this large value). This shows the importance of modelling both wake components in $3 \mathrm{D}$, and not simply assuming approximately $2 \mathrm{D}$ behaviour. 


\section{III.E. Rotor}

In order to investigate the effects of rotation on unsteady response, a single-bladed rotor with a flat-plate blade with aspect ratio 5 is studied, as shown in Figure 2(c)i. The hub radius is equal to the chord length and there is no taper. The blade is untwisted and is staggered so that the angle of attack at the blade tip is zero. The rotational speed is given in terms of the tip-speed ratio (TSR), which is the ratio of the blade tip speed to the axial flow speed. The returning wake effect is an important component of unsteady response of a rotor, and so the TSR has been set to 10 to reduce the speed at which the wake convects away from the rotor, in order to study the returning-wake interaction.

Preliminary tests at large rotation radius ( $>1$ span) showed that the reduced frequency at mid-span was a good indicator of the overall response, much like the tapered wing case (section III.C). When the spanwise interaction between sections is modelled, the response of an individual section is determined not only by the local conditions but is also affected by the behaviour of other sections. This causes local sections to tend away from their isolated 2D response and towards the global response of the whole blade, i.e. the response is equalised across the span, as noted by Namba. ${ }^{10}$ As such, it makes sense to look at the unsteady response in terms of a governing frequency, and the results of this study so far suggest that the mid-span reduced frequency is a suitable choice of governing frequency. This will be justified further in section III.F.

The gust applied is uniform, given by equation 11, acting in the axial direction. The results are compared to predictions by the Loewy function evaluated at mid-span conditions, as the Loewy function captures returning wake effects. The notation $5 \%$ span refers to the section near the rotor hub, and $95 \%$ span refers to near the tip. Figure 16 shows the load response of the rotating blade described above, plotted against the mid-span reduced frequency. There are two major effects governing the unsteady response of this blade: the reduction in aerodynamic damping at the hub compared with the tip, and the interaction with the returning wake. The mid-span response approaches $2 \mathrm{D}$ behaviour at reduced frequency 0.5 , and the Loewy function predicts the location of the peaks and troughs of the wake interaction with reasonable accuracy, although the response is slightly out of phase. However, Loewy overpredicts the magnitude of the first load peak (at reduced frequency 0.2 ) by about $20 \%$ at the mid-span, showing again the importance of including the influence of the streamwise wake and its associated aerodynamic damping.

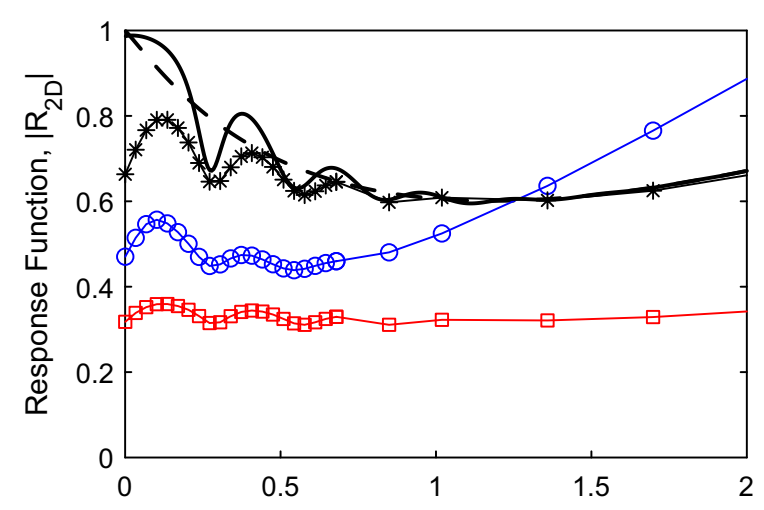

a)

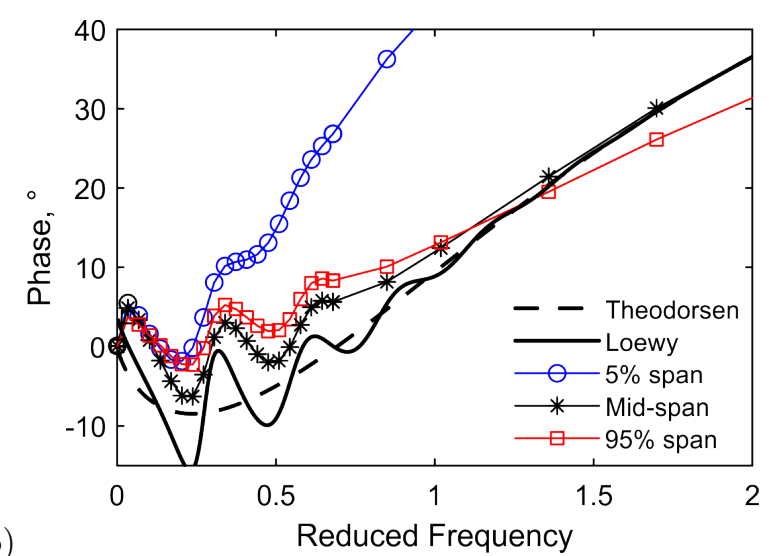

b)

Figure 16. Load response magnitude and phase of a rotating blade with an aspect ratio of 5 , rotating at TSR=10, interacting with a uniform gust acting in the axial flow direction.

Looking at the unsteady load amplitude (Figure 16(a)) at reduced frequencies below 1, it is clear that the hub and tip are experiencing more damping than the mid-span i.e. the loading is, lower at the hub and tip than at the midspan. This was also the case for non-rotating blades. In contrast to the non-rotating case, however, it can be seen that the tip experiences more damping than the hub. As the reduced frequency increases, the hub response increases rapidly, overshooting the 2D response curve. Again, this was not observed in the non-rotating cases.

Similar behaviour is seen in the phase response, although both the mid-span and the rotor tip response fall on or near the $2 \mathrm{D}$ characteristic for reduced frequencies higher than 1.0. The outboard region of a rotor 
or turbine contributes disproportionately to the torque and power output, and also to the blade bending moments. The unsteady response of this region will thus be of more significant importance to the unsteady behaviour. As such, it is of interest to note that the mid-span and tip appear to be responding in-phase, which will lead to larger fluctuations in bending moment than would be experienced if the response was out of phase between the mid-span and the tip.

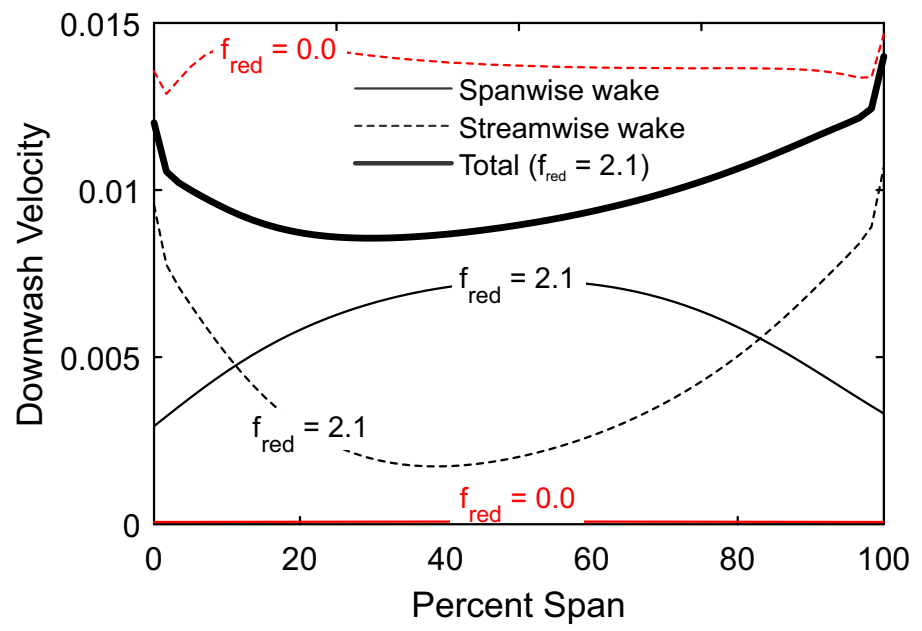

Figure 17. Spanwise distribution of downwash velocity for a flat-plate rotor blade (aspect ratio 5, TSR 10), induced by the streamwise and spanwise components of wake vorticity, for a uniform gust (eq. 11). The reduced frequency is calculated at mid-span. The downwash is normalised by the steady axial freestream velocity.

The reason for the difference between tip and hub response can be traced to the changes in downwash, which is shown in Figure 17 for mid-span reduced frequencies of 0.0 and 2.1. As in previous cases, the total downwash is strongly affected by the behaviour of both the streamwise and spanwise components of wake vorticity. The downwash induced by the spanwise wake is near-symmetrically distributed about the mid-span, because it is primarily affected by the wake being finite. The streamwise wake, on the other hand, induces a downwash that favours the tip-section of the blade, leading to a total downwash distribution that causes less aerodynamic damping near the rotor hub. This overall effect is not proportional to the steady distribution of lift, and can only be accounted for by the combined effect of unsteady spanwise and streamwise wake vorticity.

\section{III.F. Model tidal turbine}

To finish this study, the unsteady response of a model turbine is considered. The turbine geometry, shown in figure 2c)ii, has a NACA 63-series blade section with camber, twist, and tapering of the chord. The nominal operating state is TSR 4. As in the flat-plate rotor case, a uniform gust (equation 11) is applied, acting in the axial direction. The unsteady response against mid-span reduced frequency is shown in Figure 18 for a three-bladed turbine at TSR 4. The results are compared to the Loewy function, evaluated at mid-span conditions. As expected, there are fewer resonant peaks in Fig 18 than in Fig 16, showing that the reduction in TSR has reduced the wake interaction effects.

In this case, the Loewy function is a poor predictor of the load response, even at the midspan. In the region where the returning wake interaction is the strongest, over the reduced frequency range 0.0 to 0.5 , the $3 \mathrm{D}$ effects are also significant. As such, the Loewy function fails to predict the the magnitude and phase of the unsteady interaction at mid-span. There are also significant deviations from 2D response near the blade tip and hub. The changes in chord and relative flow speed across the span mean that the reduced frequency is much higher at the hub than the tip for a given mid-span reduced frequency. The response to unsteady flow at the hub is, however, relatively level across the frequency range shown and does not deviate substantially from the quasi-steady $\left(f_{\text {red }}=0\right)$ value.

As mentioned above in the flat plate rotor section (section III.E), and in the tapered blade section (section III.C), allowing blade sections to interact through 3D modelling has an equalising effect on the unsteady 


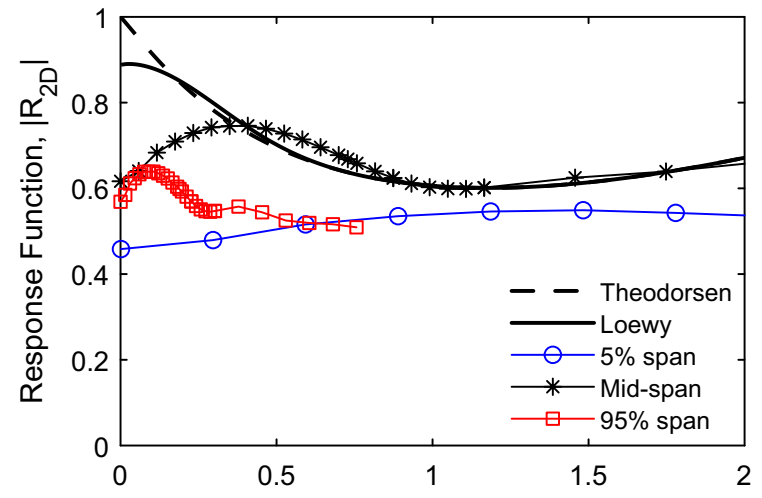

a)

Local Reduced Frequency

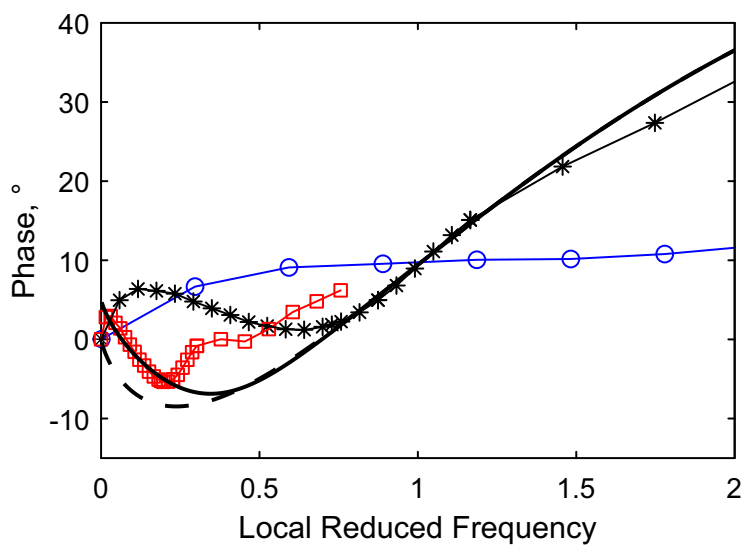

b)

Figure 18. Load response magnitude (a) and phase (b) of a 3-bladed model turbine geometry, shown in figure 2c)ii, operating at the rated TSR of 4 . The gust is uniform, acting in the axial direction. The Loewy function is evaluated at the mid-span conditions.

response, pushing the response at individual span points towards the mean response of the whole blade and reducing spanwise variations. In figure 18, the interaction between spanwise sections is causing the hub to experience much more aerodynamic damping than it would in 2D conditions at the same reduced frequency. This is an indicator of the equalising effects of the $3 \mathrm{D}$ flow, and justifies the use of a governing frequency. Having such a governing frequency for the lift response is also practical from a design perspective, as the response of a turbine to a single gust can be represented by a single vertical line on the characteristic.

To illustrate this further, Figure 19 shows the same data as in Figure 18, but this time plotted against the midspan reduced frequency. Plotting the data like this enables the response of different spanwise positions to a given gust to be compared more easily as they lie on a vertical line. The results are compared to the Loewy function, still evaluated at mid-span conditions. In contrast to the flat-plate rotor case, the phase of the tip response is close to zero (i.e. the quasi-steady phase) throughout the frequency range shown. This suggests that the downwash from the streamwise wake is significantly stronger near the tip than over the rest of the blade span. The hub section exhibits similar behaviour in the turbine and flat plate rotor cases. The amplitude of the hub response exceeds the $2 \mathrm{D}$ characteristic at reduced frequencies over 1.0 , and has an extremely large phase lead. The mid-span response exhibits $2 \mathrm{D}$ characteristics in terms of both amplitude and phase once the reduced frequency exceeds 0.8 . The reasons for this vast spanwise difference in unsteady response to a uniform gust are not clear, but it is suggested that the skew of the wake and its behaviour, particularly in the hub region, are important factors.

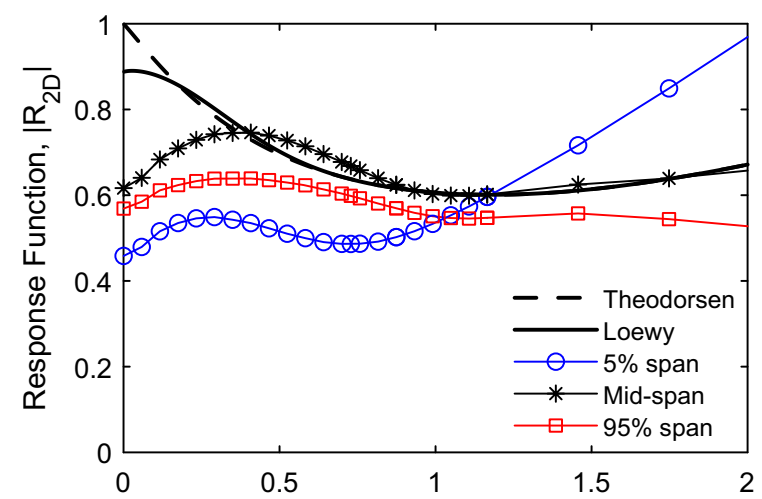

a) Midspan Reduced Frequency

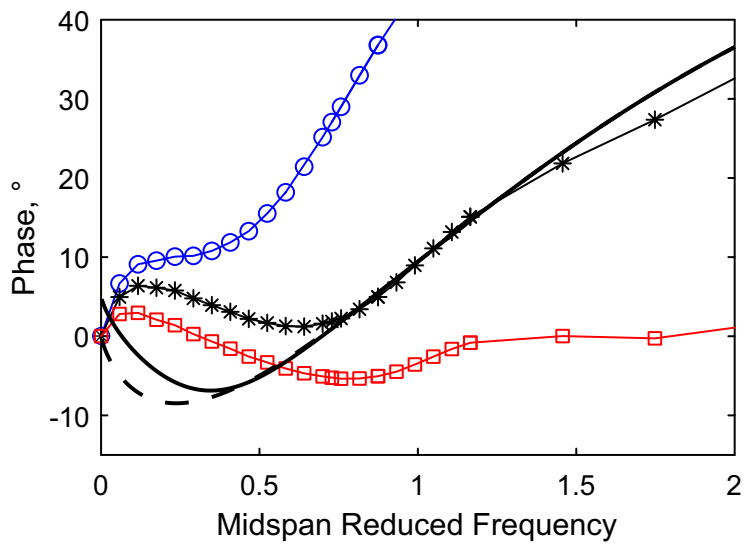

b)

Figure 19. Load response magnitude (a) and phase (b) of a 3-bladed model turbine geometry, shown in figure 2c)ii. The operating conditions are the same as in figure 18 , but here the response is plotted against the midspan reduced frequency.

The results shown above have important implications for the estimation of fatigue loads by tidal turbine 
designers. As mentioned in the introduction, Prandtl's tip loss correction is commonly applied in BEMT methods to account for the load reduction near the blade tips. Throughout this study, the lift response has generally been compared to the uncorrected 2D unsteady response function (equation 12), which at intermediate and high reduced frequencies and aspect ratios gave good estimates for the 3D load amplitude. At lower frequencies and aspect ratios, the $2 \mathrm{D}$ characteristic over-predicts the load amplitude, making it a conservative estimate. However, if correction for the steady tip-loss is applied to the $2 \mathrm{D}$ characteristic (effectively using the 3D response function, equation 13), the designer risks seriously under-predicting the unsteady load amplitude. An example of this could be seen in figure 11 (tapered blade, section III.C), where normalisation using the 3D response function produced a characteristic with larger load amplitude than the $2 \mathrm{D}$ Sears function. This suggests that if unsteady 3D effects are not accounted for, designers should apply the purely $2 \mathrm{D}$ response characteristic (equation 12), with no tip-loss corrections, for a conservative estimate of unsteady loads.

\section{Conclusions}

As a result of this study, a number of observations can be made about the effect of 3D geometry on the unsteady response of aerofoils.

Firstly, the important factors determining the level of 3D effects on an aerofoil are 1: Reduced frequency, 2: aspect ratio, and 3: proximity to tip effects. Generally, 3D effects diminish with increasing reduced frequency and increasing aspect ratio. The response near the aerofoil tip will always be more strongly influenced by $3 \mathrm{D}$ flow than that of the rest of the wing, effectively never reaching the $2 \mathrm{D}$ characteristic.

Most of the physical mechanisms governing 3D effects can be observed by studying a finite wing geometry. The magnitude and spanwise distribution of downwash plays a critical part in determining the unsteady response, since the level of downwash determines the amount of damping applied to the unsteady load. Decomposing the downwash into the contribution from spanwise and streamwise wake components enables the departures from 2D unsteady response to be understood.

The streamwise component of downwash is not considered at all in 2D analysis, while the spanwise component is assumed to extend infinitely in the spanwise direction. Both components are affected by the wing tip effects; as the tip is approached, the spanwise wake influence diminishes and the streamwise wake influence increases. At intermediate reduced frequencies and aspect ratios, both components of wake vorticity contribute to the total downwash at mid-span, but for the case of a finite wing they still sum to form a downwash approximately equal to the $2 \mathrm{D}$ load amplitude. This is despite the individual components having sinificant 3D characteristics. For more complex geometries (sweep, taper, rotation), these two components do not always add up to the 2D response. This emphasises the importance, from a modelling perspective, of including both wake components in 3D analysis.

The phase of the load response was observed to vary significantly along the span, leading to different spanwise sections responding out of phase with each other even with no spanwise variation in gust shape. This has particular importance to any application where the load is integrated along the blade span, for example the evaluation of blade bending moment. This finding therefore has significant implications for the prediction of the fatigue life of tidal turbines, since spanwise phase differences will reduce the total load fluctuation on the blade.

The importance of considering the correct non-dimensional gust frequency and response amplitude has been shown. In the case of geometry variations which cause a spanwise variation in reduced frequency (e.g taper), the spanwise variations in the amplitude and phase of the response will collapse onto one line when

plotted against local reduced frequency. There is no equivalent method for collapsing the results for geometry features which induce spanwise flow variations without changing the spanwise variation in reduced frequency (e.g. sweep).

The Loewy function was found to predict the location of the peaks and troughs of the returning wake interaction fairly well for a flat-plate rotor blade, but it failed to capture the unsteady response of a model 
tidal turbine with realistic camber, twist and taper. For the tidal turbine, the unsteady wake interaction is strongest in a frequency range where 3D effects are significant, and so the amplitude and phase were not well predicted by 2D theory. For both the flat-plate rotor and the tidal turbine, the hub response deviates strongly from the $2 \mathrm{D}$ characteristic, and is much less affected by aerodynamic damping than the rest of the span. The tip response of the turbine was near quasi-steady over the range considered, due to it experiencing more aerodynamic damping than the rest of the span. The mid-span reduced frequency was generally used to indicate the response across the whole span. The use of the mid-span frequency as the governing frequency is justified by the equalising effect of the $3 \mathrm{D}$ flow system, and is practical from a design perspective.

Finally, if a designer is neglecting 3D effects when making unsteady load calculations, a conservative estimate of the unsteady load amplitude can be obtained by using the $2 \mathrm{D}$ response characteristic, although this would not give an accurate estimate of the phase response along the span. The $2 \mathrm{D}$ characteristic must however be applied with no added steady tip-loss corrections to remain a conservative estimate. Accounting for only the steady tip-loss effects will lead to significant under-prediction of the unsteady load amplitude.

\section{References}

\footnotetext{
${ }^{1}$ Burton, T., Sharpe, D., Jenkins, N., Bossanyi, E., Wind Energy Handbook, John Wiley \& Sons, 2001.

${ }^{2}$ Bossanyi, E., GH Tidal Bladed Theory Manual, 2007.

${ }^{3}$ Leishman, J. G.Principles of Helicopter Aerodynamics, Chapter 8, Cambridge University Press, 2006.

${ }^{4}$ Theodorsen, T. General theory of aerodynamic instability and the mechanism of flutter], NACA Techical Report no. 496, 1935.

${ }^{5}$ Sears, W.A Systematic Presentation of the Theory of Thin Aerofoils in Non-Uniform Motion, Thesis, California Institute of Technology, 1938 .

${ }^{6}$ Leishman, J.G., Challenges in Modelling the Unsteady Aerodynamics of Wind Turbines, Wind Energy, 2002.

${ }^{7}$ Winter, A., Differences in fundamental design drivers for wind and tidal turbines, Proceedings of IEEE OCEANS, 2011.

${ }^{8}$ Clark, T. H. E., Turbulence in Marine Environments (TiME): A framework for understanding turbulence and its effects on tidal devices, Proceedings of the 11th European Wave and Tidal Energy Conference, 2015.

${ }^{9}$ Namba, M., Toshimitsu, K., Double Linearisation Theory of Three-Dimensional Cascades With Vibrating Blades Under Spanwise-Nonuniform Mean Loading, I: Subsonic Flow, Journal of Sound and Vibration, 1991.

${ }^{10}$ Namba, M., Three-dimensional flow, AGARD Manual on Aeroelasticity in Axial-FLow Turbomachines Volume 1: Unsteady Turbomachinery Aerodynamics, chapter 4, $198 \%$.

${ }^{11}$ Schulten, J.B.H.M., Sound Generated by Rotor Wakes Interacting with a Leaned Vane Stator, AIAA Journal, 1982.

${ }^{12}$ Hall, K. C., Lorence, C. B., Calculation of Three-Dimensional Unsteady Flows in Turbomachinery Using the Linearized Harmonic Euler Equations, ASME, 1992.

${ }^{13}$ Verdon, J.M., Linearized Unsteady Aerodynamic Analysis of the Acoustic Response to Wake / Blade-Row Interaction, NASA Contractor Report 2001-210713, 2001.

${ }^{14}$ Golubev, V., Atassi, H., Lipatov, A., 3-D unsteady effects in annular cascades with swirl and comparison with 2-D strip theory, 3rd AIAA/CEAS Aeroacoustics Conference, 1997.

${ }^{15}$ Scarlett, G.T., Sellar, B., van den Bremer, T., Viola, I.M., Unsteady Hydrodynamics of a Full-Scale Tidal Turbine, 7th European Conference on Computational Fluid Dynamics (ECFD 7), 2018.

${ }^{16}$ Milne, I. A., Day, A. H., Sharma, R. N., Flay, R. G. J., Blade loads on tidal turbines in planar oscillatory flow, Ocean Engineering, 2013.

${ }^{17}$ McNae, D., Unsteady Hydrodynamics of Tidal Stream Turbines, thesis, Imperial College London, 2013.

${ }^{18}$ Katz, J., Plotkin, A., Low-Speed Aerodynamics, Cambridge University Press, 2001.

${ }^{19}$ Kinnas, S. A., Hsin, C. Boundary Element Method for the Analysis of the Unsteady Flow Around Extreme Propeller Geometries, AIAA Journal, 1992.

${ }^{20}$ Sequeira, C. L., Miller, R. J., Unsteady Gust Response of Tidal Stream Turbines, In Proceedings of the IEEE/MTS OCEANS, 2014.

${ }^{21}$ Sequeira, C. L., Hydrodynamics of Tidal Stream Turbines, Thesis, Cambridge University, 2014.

${ }^{22}$ Loewy, R. G., A two-dimensional approximation to the unsteady aerodynamics of rotary wings, Journal of the Aeronautical Sciences, $195 \%$.

${ }^{23}$ Young, A. M., Farman, J. R., Miller, R. J.,Load alleviation technology for extending life in tidal turbines, Progress in Renewable Energies Offshore-Proceedings of 2nd International Conference on Renewable Energies Offshore, RENEW 2016.

${ }^{24}$ Caradonna, F. X., Tung, C., Experimental and Analytical Studies of a Model Helicopter Rotor in Hover, NASA Technical Memorandum 81232, 1981.

${ }^{25}$ Tan, J., Wang, H., Simulating unsteady aerodynamics of helicopter rotor with panel/viscous vortex particle method, Aerospace Science and Technology, 2013.
} 